\title{
STRUCTURE OF APPROXIMATE SOLUTIONS OF VARIATIONAL PROBLEMS WITH EXTENDED-VALUED CONVEX INTEGRANDS
}

\author{
Alexander J. Zaslavski ${ }^{1}$
}

\begin{abstract}
In this work we study the structure of approximate solutions of autonomous variational problems with a lower semicontinuous strictly convex integrand $f: R^{n} \times R^{n} \rightarrow R^{1} \cup\{\infty\}$, where $R^{n}$ is the $n$-dimensional Euclidean space. We obtain a full description of the structure of the approximate solutions which is independent of the length of the interval, for all sufficiently large intervals.
\end{abstract}

Mathematics Subject Classification. 49J99.

Received June 6, 2007. Revised May 1st, 2008.

Published online August 20, 2008.

\section{INTRODUCTION}

In this paper we study the structure of approximate solutions of the following variational problem

$$
\int_{0}^{T} f\left(v(t), v^{\prime}(t)\right) \mathrm{d} t \rightarrow \min
$$

$$
v:[0, T] \rightarrow R^{n} \text { is an absolutely continuous (a.c.) function such that } v(0)=x,
$$

where $x \in R^{n}$ and $T>0$. Here $R^{n}$ is the $n$-dimensional Euclidean space with the Euclidean norm $|\cdot|$ and $f: R^{n} \times R^{n} \rightarrow R^{1} \cup\{\infty\}$ is an extended-valued integrand.

We are interested in a turnpike property of the approximate solutions of the problem $(\mathrm{P})$ which is independent of the length of the interval $T$, for all sufficiently large intervals. To have this property means, roughly speaking, that the approximate solutions of the variational problems are determined mainly by the integrand $f$, and are essentially independent of $T$ and $x$.

Turnpike properties are well known in mathematical economics. The term was first coined by Samuelson in 1948 (see [13]) where he showed that an efficient expanding economy would spend most of the time in the vicinity of a balanced equilibrium path (also called a von Neumann path). This property was further investigated for optimal trajectories of models of economic dynamics (see, for example, $[1,3,8,14,17]$ and the references mentioned there). In the classical turnpike theory the function $f$ has the turnpike property (TP) if there exists $\bar{x} \in R^{n}$ (a turnpike) which satisfies the following condition:

\footnotetext{
Keywords and phrases. Good function, infinite horizon, integrand, overtaking optimal function, turnpike property.

1 Department of Mathematics, The Technion-Israel Institute of Technology, 32000 Haifa, Israel. ajzasltx.technion.ac.il
} 
For each $M, \epsilon>0$ there is a natural number $L$ such that for each number $T \geq 2 L$, each $x \in R^{n}$ satisfying $|x| \leq M$ and each solution $v:[0, T] \rightarrow R^{n}$ of the problem $(\mathrm{P})$ the inequality $|v(t)-\bar{x}| \leq \epsilon$ holds for all $t \in[L, T-L]$.

Note that $L$ depends neither on $T$ nor on $x$.

In the classical turnpike theory $[1,3,8,14]$ the cost function $f$ is strictly convex. Under this assumption the turnpike property can be established and the turnpike $\bar{x}$ is a unique solution of the minimization problem $f(x, 0) \rightarrow \min , x \in R^{n}$. In this situation it is shown that for each a.c. function $v:[0, \infty) \rightarrow R^{n}$ either the function

$$
T \rightarrow \int_{0}^{T} f\left(v(t), v^{\prime}(t)\right) \mathrm{d} t-T f(\bar{x}, 0), T \in(0, \infty)
$$

is bounded (in this case the function $v$ is called $(f)$-good) or it diverges to $\infty$ as $T \rightarrow \infty$. Moreover, it is also established that any $(f)$-good function converges to the turnpike $\bar{x}$. In the sequel this property is called the asymptotic turnpike property.

Recently it was shown that the turnpike property is a general phenomenon which holds for large classes of variational and optimal control problems without convexity assumptions. (See, for example, [7,15-17] and the references mentioned therein.) For these classes of problems a turnpike is not necessarily a singleton but may instead be a nonstationary trajectory (in the discrete time nonautonomous case) or an absolutely continuous function on the interval $[0, \infty)$ (in the continuous time nonautonomous case) or a compact subset of the space $R^{n}$ (in the autonomous case). Note that all of these recent results were obtained for finite-valued integrands $f$ (in other words, for unconstrained variational problems). In this paper we study the problems (P) with an extended-valued integrand $f: R^{n} \times R^{n} \rightarrow R^{1} \cup\{\infty\}$ (in other words, constrained variational problems). Clearly, these constrained problems with extended-valued integrands are more difficult and less understood than their unconstrained prototypes in [15-18]. They are also more realistic from the point of view of applications. As we have mentioned before in general a turnpike is not necessarily a singleton. Nevertheless problems of the type $(\mathrm{P})$ for which the turnpike is a singleton are of great importance because of the following reasons: there are many models for which a turnpike is a singleton; if a turnpike is a singleton, then approximate solutions of (P) have very simple structure and this is very important for applications; if a turnpike is a singleton, then it can be easily calculated as a solution of the problem $f(x, 0) \rightarrow \min , x \in R^{n}$.

In our recent paper [19] the goal is to understand when the turnpike property holds with the turnpike being a singleton. We show there that the turnpike property follows from the asymptotic turnpike property. More precisely, we assume that any $(f)$-good function converges to a unique solution $\bar{x}$ of the problem $f(x, 0) \rightarrow$ min, $x \in R^{n}$ and show that the turnpike property holds and $\bar{x}$ is the turnpike (see [19], Thm. 1.1). Note that in [19] we do not use convexity assumptions. It should be mentioned that analogous results which show that turnpike properties follow from asymptotic turnpike properties for unconstrained variational problems with finite-valued integrands were obtained in $[7,17]$.

The goal of the present paper is to study the structure of approximate solutions of the problems $(\mathrm{P})$ in the regions $[0, L]$ and $[T-L, T]$ (see the definition of the turnpike property). We will show (see Thm. 3.2) that if $v:[0, T] \rightarrow R^{n}$ is an approximate solution of the problem $(\mathrm{P})$, then for all $t \in[0, L]$ the state $v(t)$ is arbitrary close to $X(t)$ where $X:[0, \infty) \rightarrow R^{n}$ is a unique solution of a certain infinite horizon optimal control problem satisfying $X(0)=x$. We will also show (see Thm. 3.3) that if $v:[0, T] \rightarrow R^{n}$ is an approximate solution of the problem $(\mathrm{P})$, then for all $t \in[0, L]$ the state $v(T-t)$ is arbitrary close to $\Lambda(t)$, where $\Lambda:[0, \infty) \rightarrow R^{n}$ is a unique solution of a certain infinite horizon optimal control problem which does not depend on $x$. These results are established when the function $f$ is strictly convex. In this case combining Theorem 1.1 of [19] and Theorems 3.2 and 3.3 of the present paper we obtain the full description of the structure of approximate solutions of the problems $(\mathrm{P})$. Note that the structure of approximate solutions of the problems $(\mathrm{P})$ in the region $[0, L]$ depends on $x$ while their structure in the region $[T-L, T]$ does not depend on $x$. Actually it depends only on $f$ and we have here a new kind of the turnpike property. 


\section{Preliminaries}

In this paper we denote by $\operatorname{mes}(E)$ the Lebesgue measure of a Lebesgue measurable set $E \subset R^{1}$, denote by $|\cdot|$ the Euclidean norm of the $n$-dimensional space $R^{n}$ and by $\langle\cdot, \cdot\rangle$ the inner product of $R^{n}$. For each function $h: X \rightarrow R^{1} \cup\{\infty\}$, where $X$ is nonempty, set $\operatorname{dom}(h)=\{x \in X: h(x)<\infty\}$.

Let $a>0, \psi:[0, \infty) \rightarrow[0, \infty)$ be an increasing function which satisfies

$$
\lim _{t \rightarrow \infty} \psi(t)=\infty
$$

and let $f: R^{n} \times R^{n} \rightarrow R^{1} \cup\{\infty\}$ be a convex lower semicontinuous function such that the $\operatorname{set} \operatorname{dom}(f)$ is nonempty and closed and that

$$
f(x, y) \geq \max \{\psi(|x|), \psi(|y|)|y|\}-a \text { for each } x, y \in R^{n} .
$$

We suppose that there exists $\bar{x} \in R^{n}$ such that the following assumption holds:

(A1) $(\bar{x}, 0)$ is an interior point of the set $\operatorname{dom}(f)$ and

$$
f(\bar{x}, 0) \leq f(x, 0) \text { for all } x \in R^{n}
$$

Remark 2.1. Note that the existence of $\bar{x} \in R^{n}$ satisfying (2.3) follows from (2.1) and (2.2). In this paper we also assume that $(\bar{x}, 0)$ is an interior point of the set $\operatorname{dom}(f)$.

They are well-known facts from convex analysis [12] that the function $f$ is continuous at the point $(\bar{x}, 0)$ and that there is $l \in R^{n}$ such that

$$
f(x, y) \geq f(\bar{x}, 0)+\langle l, y\rangle \text { for each } x, y \in R^{n} .
$$

We also assume that for each pair $\left(x_{1}, y_{1}\right),\left(x_{2}, y_{2}\right) \in \operatorname{dom}(f)$ such that $\left(x_{1}, y_{1}\right) \neq\left(x_{2}, y_{2}\right)$ and each $\alpha \in(0,1)$ the inequality

$$
f\left(\alpha\left(x_{1}, y_{1}\right)+(1-\alpha)\left(x_{2}, y_{2}\right)\right)<\alpha f\left(x_{1}, y_{1}\right)+(1-\alpha) f\left(x_{2}, y_{2}\right)
$$

holds. This means that the function $f$ is strictly convex. The integrand $f$ was considered in [19], Example 2 . It was shown there that all the results of [19] hold for the integrand $f$.

In our study we will use an integrand $L$ defined by

$$
L(x, y)=f(x, y)-f(\bar{x}, 0)-\langle l, y\rangle \text { for all } x, y \in R^{n} .
$$

We consider the following variational problem

$$
\begin{gathered}
\int_{0}^{T} f\left(v(t), v^{\prime}(t)\right) \mathrm{d} t \rightarrow \min , \\
v:[0, T] \rightarrow R^{n} \text { is an a.c. function such that } v(0)=x,
\end{gathered}
$$

where $x \in R^{n}$ and $T>0$.

For each $x \in R^{n}$ and each number $T>0$ set

$$
\begin{gathered}
\sigma(f, T, x)=\inf \left\{\int_{0}^{T} f\left(v(t), v^{\prime}(t)\right) \mathrm{d} t: v:[0, T] \rightarrow R^{n} \text { is an a.c. function satisfying } v(0)=x\right\}, \\
\sigma(f, T)=\inf \left\{\int_{0}^{T} f\left(v(t), v^{\prime}(t)\right) \mathrm{d} t: v:[0, T] \rightarrow R^{n} \text { is an a.c. function }\right\} .
\end{gathered}
$$


For each $T_{1} \in R^{1}, T_{2}>T_{1}$ and each a.c. function $v:\left[T_{1}, T_{2}\right] \rightarrow R^{n}$ set

$$
I^{f}\left(T_{1}, T_{2}, v\right)=\int_{T_{1}}^{T_{2}} f\left(v(t), v^{\prime}(t)\right) \mathrm{d} t
$$

In [19] we study a class of integrands which contains the integrand $f$ and obtain the following useful results.

Proposition 2.1 ([19], Prop. 7.1). For each $M>0$ there exists $c_{M}>0$ such that

$$
\sigma(f, T, x) \geq T f(\bar{x}, 0)-c_{M}
$$

for each $x \in R^{n}$ satisfying $|x| \leq M$ and each $T>0$.

By Proposition 2.1 for each a.c. function $v:[0, \infty) \rightarrow R^{n}$ the function

$$
T \rightarrow \int_{0}^{T} f\left(v(t), v^{\prime}(t)\right) \mathrm{d} t-T f(\bar{x}, 0), T \in(0, \infty)
$$

is bounded from below.

We say that an a.c. function $v:[0, \infty) \rightarrow R^{n}$ is called $(f) \operatorname{good}[3,5,17]$ if

$$
\sup \left\{\left|\int_{0}^{T} f\left(v(t), v^{\prime}(t)\right) \mathrm{d} t-T f(\bar{x}, 0)\right|: T \in(0, \infty)\right\}<\infty .
$$

It should be mentioned that our study of the structure of solutions of variational problems on intervals $[0, T]$ with sufficiently large length $T$ is strongly based on asymptotic behavior of $(f)$-good functions [17].

Proposition 2.2 ([19], Prop. 1.1). Let $v:[0, \infty) \rightarrow R^{n}$ be an a.c. function. Then either $v$ is $(f)$-good or

$$
\int_{0}^{T} f\left(v(t), v^{\prime}(t)\right) \mathrm{d} t-T f(\bar{x}, 0) \rightarrow \infty \text { as } T \rightarrow \infty .
$$

Moreover, if $v$ is $(f)$-good, then $\sup \{|v(t)|: t \in[0, \infty)\}<\infty$.

Proposition 2.3 ([19], Prop. 7.2). Let $v:[0, \infty) \rightarrow R^{n}$ be an $(f)$-good function. Then

$$
\lim _{t \rightarrow \infty}|v(t)-\bar{x}|=0
$$

For each $M>0$ denote by $X_{M}$ the set of all $x \in R^{n}$ such that $|x| \leq M$ and that there exists an a.c. function $v:[0, \infty) \rightarrow R^{n}$ which satisfies

$$
v(0)=x, I^{f}(0, T, v)-T f(\bar{x}, 0) \leq M \text { for each } T \in(0, \infty) .
$$

The following turnpike result was established in [19], Theorem 1.1.

Theorem 2.1. Let $\epsilon, M>0$. Then there exist a natural number $L$ and a positive number $\delta$ such that for each real $T>2 L$ and each a.c. function $v:[0, T] \rightarrow R^{n}$ which satisfies

$$
v(0) \in X_{M} \text { and } I^{f}(0, T, v) \leq \sigma(f, T, v(0))+\delta
$$

there exist $\tau_{1} \in[0, L]$ and $\tau_{2} \in[T-L, T]$ such that

$$
|v(t)-\bar{x}| \leq \epsilon \text { for all } t \in\left[\tau_{1}, \tau_{2}\right]
$$

and if $|v(0)-\bar{x}| \leq \delta$, then $\tau_{1}=0$. 
In the sequel we use a notion of an overtaking optimal function introduced in [1,3,14].

An a.c. function $v:[0, \infty) \rightarrow R^{n}$ is called $(f)$-overtaking optimal if for each a.c. function $u:[0, \infty) \rightarrow R^{n}$ satisfying $u(0)=v(0)$,

$$
\limsup _{T \rightarrow \infty}\left[I^{f}(0, T, v)-I^{f}(0, T, u)\right] \leq 0 .
$$

The following result obtained in [19], Theorem 1.2, establishes the existence of an overtaking optimal function.

Theorem 2.2. Assume that $x \in R^{n}$ and that there exists an $(f)$-good function $v:[0, \infty) \rightarrow R^{n}$ satisfying $v(0)=x$. Then there exists an $(f)$-overtaking optimal function $u_{*}:[0, \infty) \rightarrow R^{n}$ such that $u_{*}(0)=x$.

The following optimality notion is also used in the study of infinite horizon variational problems (see $[4,6,7,9-11]$ and the references mentioned there).

An a.c. function $v:[0, \infty) \rightarrow R^{n}$ is called $(f)$-minimal if for each $T_{1} \geq 0$, each $T_{2}>T_{1}$ and each a.c. function $u:\left[T_{1}, T_{2}\right] \rightarrow R^{n}$ satisfying $u\left(T_{i}\right)=v\left(T_{i}\right), i=1,2$ the inequality

$$
\int_{T_{1}}^{T_{2}} f\left(v(t), v^{\prime}(t)\right) \mathrm{d} t \leq \int_{T_{1}}^{T_{2}} f\left(u(t), u^{\prime}(t)\right) \mathrm{d} t
$$

holds.

We will show in our forthcoming paper that an a.c. function $v:[0, \infty) \rightarrow R^{n}$ is $(f)$-overtaking optimal if and only if $v$ is $(f)$-minimal and $(f)$-good.

\section{MAin RESUlts}

In this section we state our main results which describe the structure of approximate solutions of variational problems in the regions containing end points. We consider the variational problems with the integrand $f$ introduced in Section 2. We suppose that all the assumptions posed in Section 2 hold. In addition we suppose that the following assumption holds.

(A2) For each $M, \epsilon>0$ there exists $\gamma>0$ such that for each pair of points $\left(\xi_{1}, \xi_{2}\right),\left(\eta_{1}, \eta_{2}\right) \in \operatorname{dom}(f)$ which satisfies $\left|\xi_{i}\right|,\left|\eta_{i}\right| \leq M, i=1,2$ and $\left|\xi_{1}-\xi_{2}\right| \geq \epsilon$ the following inequality holds:

$$
2^{-1} f\left(\xi_{1}, \eta_{1}\right)+2^{-1} f\left(\xi_{2}, \eta_{2}\right)-f\left(2^{-1}\left(\xi_{1}+\xi_{2}\right), 2^{-1}\left(\eta_{1}+\eta_{2}\right)\right) \geq \gamma .
$$

Remark 3.1. Note that (A2) follows from (2.5) if the restriction of $f$ to $\operatorname{dom}(f)$ is continuous.

Since the restriction of $f$ to $\operatorname{dom}(f)$ is strictly convex (see (A2)) Theorem 2.2 implies the following result.

Theorem 3.1. Assume that $x \in R^{n}$ and that there exists an $(f)$-good function $v:[0, \infty) \rightarrow R^{n}$ satisfying $v(0)=x$. Then there exists a unique $(f)$-overtaking optimal function $v_{*}:[0, \infty) \rightarrow R^{n}$ such that $v_{*}(0)=x$.

Let $z \in R^{n}$ and let there exist an $(f)$-good function $v:[0, \infty) \rightarrow R^{n}$ such that $v(0)=z$. Denote by $Y^{(f, z)}$ : $[0, \infty) \rightarrow R^{n}$ a unique $(f)$-overtaking optimal function satisfying $Y^{(f, z)}(0)=z$ which exists by Theorem 3.1.

In the following theorem (as in the whole section) we suppose that assumptions (A1) and (A2) hold. This theorem which will be proved in Section 5 describes the structure of approximate solutions of variational problems in the regions containing the left end point.

Theorem 3.2. Let $M, \epsilon$ be positive numbers and let $L_{0}$ be a natural number. Then there exist $\delta>0$ and a natural number $L_{1}>L_{0}$ such that for each number $T \geq L_{1}$, each $z \in X_{M}$ and each a.c. function $v:[0, T] \rightarrow R^{n}$ which satisfies

the following inequality holds:

$$
v(0)=z, I^{f}(0, T, v) \leq \sigma(f, T, z)+\delta
$$

$$
\left|v(t)-Y^{(f, z)}(t)\right| \leq \epsilon, t \in\left[0, L_{0}\right] .
$$


Now we intend to describe the structure of approximate solutions of variational problems in the regions containing the right end point. In order to meet this goal define the functions $\bar{f}, \bar{L}: R^{n} \times R^{n} \rightarrow R^{1} \cup\{\infty\}$ by

$$
\bar{f}(x, y)=f(x,-y), \bar{L}(x, y)=L(x,-y) \text { for all } x, y \in R^{n} .
$$

It is clear that

$$
\operatorname{dom}(\bar{f})=\left\{(x, y) \in R^{n} \times R^{n}:(x,-y) \in \operatorname{dom}(f)\right\}
$$

$\operatorname{dom}(\bar{f})$ is nonempty closed convex subset of $R^{n} \times R^{n}$,

$$
\bar{f}(x, y) \geq \max \{\psi(|x|), \psi(|y|)|y|\}-a \text { for each } x, y \in R^{n} \times R^{n}
$$

$(\bar{x}, 0)$ is an interior point of the set $\operatorname{dom}(\bar{f})$ and the function $\bar{f}$ is convex and lower semicontinuous.

By (3.1), (2.4) and (2.6) for each $x, y \in R^{n}$

$$
\begin{gathered}
\bar{f}(x, y)=f(x,-y) \geq f(\bar{x}, 0)+\langle l,-y\rangle=\bar{f}(\bar{x}, 0)+\langle-l, y\rangle, \\
\bar{L}(x, y)=L(x,-y)=f(x,-y)-f(\bar{x}, 0)-\langle l,-y\rangle=\bar{f}(x, y)-\bar{f}(\bar{x}, 0)-\langle-l, y\rangle .
\end{gathered}
$$

In view of $(3.1),(3.2)$ and $(2.5)$ for each $\left(x_{1}, y_{1}\right),\left(x_{2}, y_{2}\right) \in \operatorname{dom}(\bar{f})$ such that $\left(x_{1}, y_{1}\right) \neq\left(x_{2}, y_{2}\right)$ and each $\alpha \in(0,1)$

$$
\bar{f}\left(\alpha\left(x_{1}, y_{1}\right)+(1-\alpha)\left(x_{2}, y_{2}\right)\right)<\alpha \bar{f}\left(x_{1}, y_{1}\right)+(1-\alpha) \bar{f}\left(x_{2}, y_{2}\right)
$$

Therefore all the assumptions posed in Section 2 for the function $f$ also hold for the function $\bar{f}$. Also all the results of Section 2 stated for the function $f$ are valid for the function $\bar{f}$. In particular Theorems 2.1 and 2.2 hold for the integrand $\bar{f}$.

Assumption (A2) and (3.1) imply that the following assumption holds.

(A3) For each pair of positive numbers $M, \epsilon$ there exists $\gamma>0$ such that for each pair $\left(\xi_{1}, \xi_{2}\right),\left(\eta_{1}, \eta_{2}\right) \in$ $\operatorname{dom}(\bar{f})$ which satisfies

$$
\left|\xi_{i}\right|,\left|\eta_{i}\right| \leq M, i=1,2 \text { and }\left|\xi_{1}-\xi_{2}\right| \geq \epsilon
$$

the inequality

$$
2^{-1} \bar{f}\left(\xi_{1}, \eta_{1}\right)+2^{-1} \bar{f}\left(\xi_{2}, \eta_{2}\right)-\bar{f}\left(2^{-1}\left(\xi_{1}+\xi_{2}\right), 2^{-1}\left(\eta_{1}+\eta_{2}\right)\right) \geq \gamma_{0}
$$

It is clear now that Theorems 3.1 and 3.2 hold for the integrand $\bar{f}$.

For each $M>0$ denote by $\bar{X}_{M}$ the set of all $x \in R^{n}$ such that $|x| \leq M$ and that there exists an a.c. function $v:[0, \infty) \rightarrow R^{n}$ which satisfies

$$
I^{\bar{f}}(0, T, v)-T \bar{f}(\bar{x}, 0) \leq M \text { for each } T \in(0, \infty)
$$

Set

$$
\bar{X}_{*}=\cup\left\{\bar{X}_{M}: M \in(0, \infty)\right\} .
$$

Since the function $\bar{f}$ is convex we obtain that the set $\bar{X}_{M}$ is convex for all $M>0$. In view of Proposition 2.2 of [19] (see also Prop. 4.1) for each $M>0$ the set $\bar{X}_{M}$ is closed.

It follows from Theorem 3.1 applied to the integrand $\bar{f}$ that for each $x \in \bar{X}_{*}$ there exists a unique $(\bar{f})$ overtaking optimal function $\Lambda^{(x)}:[0, \infty) \rightarrow R^{n}$ such that $\Lambda^{(x)}(0)=x$. In view of Proposition $2.2 \Lambda^{(x)}$ is $(\bar{f})$-good for any $x \in \bar{X}_{*}$. Proposition 2.3 implies that for each $x \in \bar{X}_{*}$

$$
\lim _{t \rightarrow \infty}\left|\Lambda^{(x)}(t)-\bar{x}\right|=0
$$

For each $x \in \bar{X}_{*}$ put

$$
\pi(x)=\lim _{T \rightarrow \infty}\left[I^{\bar{f}}\left(0, T, \Lambda^{(x)}\right)-T \bar{f} \overline{(x, 0)}\right] .
$$


Let $x \in \bar{X}_{*}$. We show that $\pi(x)$ is well-defined and finite. By (3.5), (3.9) and (3.10)

$$
\begin{aligned}
\pi(x) & =\lim _{T \rightarrow \infty}\left[\int_{0}^{T} \bar{L}\left(\Lambda^{(x)}(t),\left(\Lambda^{(x)}\right)^{\prime}(t)\right) \mathrm{d} t-\int_{0}^{T}\left\langle l,\left(\Lambda^{(x)}\right)^{\prime}(t)\right\rangle \mathrm{d} t\right] \\
& =\lim _{T \rightarrow \infty} \int_{0}^{T} \bar{L}\left(\Lambda^{(x)}(t),\left(\Lambda^{(x)}\right)^{\prime}(t)\right) \mathrm{d} t-\lim _{T \rightarrow \infty}\left\langle l, \Lambda^{(x)}(T)-x\right\rangle \\
& =\int_{0}^{\infty} \bar{L}\left(\Lambda^{(x)}(t),\left(\Lambda^{(x)}\right)^{\prime}(t)\right) \mathrm{d} t-\langle l, \bar{x}-x\rangle .
\end{aligned}
$$

Thus $\pi(x)$ is well-defined. Since $\Lambda^{(x)}$ is $(\bar{f})$-good Proposition 2.2 implies that $\pi(x)$ is finite for each $x \in \bar{X}_{*}$.

The function $\pi$ plays an important role in our study of the structure of approximate solutions of variational problems in the regions containing the right end point. We will show that approximate solutions of the problem $(\mathrm{P})$ are arbitrary close to the function $\Lambda^{\left(x_{*}\right)}(T-t)$ in a region which contains the right end point $T$, where $x_{*}$ is a unique point of minimum of the function $\pi$.

In Section 6 we will prove the following result.

\section{Proposition 3.1.}

1. For each $M>0$ the function $\pi: \bar{X}_{M} \rightarrow R^{1}$ is lower semicontinuous.

2. For all $y, z \in \bar{X}_{*}$ satisfying $y \neq z$ and each $\alpha \in(0,1)$,

$$
\pi(\alpha y+(1-\alpha) z)<\alpha \pi(y)+(1-\alpha) \pi(z) .
$$

3. $\pi(\bar{x})=0$.

4. There is $\tilde{M}>|\bar{x}|$ such that $\pi(x) \geq 2$ for each $x \in \bar{X}_{*} \backslash \bar{X}_{\tilde{M}}$.

Let $\tilde{M}>0$ be as guaranteed by Proposition 3.1. It follows from Proposition 3.1 that there exists a unique $x_{*} \in \bar{X}_{\tilde{M}}$ such that

In view of Proposition 3.1 if $x \in \bar{X}_{*} \backslash \bar{X}_{\tilde{M}}$, then

$$
\pi\left(x_{*}\right)<\pi(x) \text { for all } x \in \bar{X}_{\tilde{M}} \backslash\left\{x_{*}\right\} .
$$

$$
\pi(x) \geq 2>\pi(\bar{x}) \geq \pi\left(x_{*}\right) .
$$

In the following theorem (as in the whole section) we suppose that assumptions (A1) and (A2) hold. This theorem describes the structure of approximate solutions of variational problems in the regions containing the right end point.

Theorem 3.3. Let $M, \epsilon$ be positive numbers and let $L_{1}$ be a natural number. Then there exist $\delta>0$ and a natural number $L_{2}>L_{1}$ such that if a real number $T>2 L_{2}$ and if an a.c. function $v:[0, T] \rightarrow R^{n}$ satisfies

$$
v(0) \in X_{M} \text { and } I^{f}(0, T, v) \leq \sigma(f, T, v(0))+\delta,
$$

then

$$
\left|v(T-t)-\Lambda^{\left(x_{*}\right)}(t)\right| \leq \epsilon \text { for all } t \in\left[0, L_{1}\right] .
$$

Theorem 3.3 will be proved in Section 7 .

Note that one can easily construct a broad class of integrands satisfying the assumptions posed in the paper and for which our results hold. For example, assume that $K$ is a closed convex subset of $R^{n} \times R^{n}$ with a nonempty interior and $f: K \rightarrow R^{1}$ is a strictly convex continuous function for which the minimization problem $f(x, 0) \rightarrow$ min subject to $(x, 0) \in K$ has a solution $\bar{x}$ such that $(\bar{x}, 0)$ is an interior point of $K$ and such that $f(x, y) \geq c_{1}|x|+c_{2}|y|^{p}-c_{3}$ for all $(x, y) \in R^{n} \times R^{n}$, where $c_{1}, c_{2}, c_{3}>0$ and $p>1$ are constants. We set 
$f(x, y)=\infty$ for all $(x, y) \in R^{2 n} \backslash K$. It is not difficult to see that the integrand $f$ satisfies all the assumptions posed in Sections 1 and 2 and Theorems 3.1-3.3 hold for $f$.

The characterization of approximate solutions in the initial and final periods is implicit: it is in terms of unique $(f)$-overtaking functions satisfying certain boundary conditions. In order to obtain approximations of these $(f)$-overtaking functions we need to find a finite number of approximate solutions of the problem $(\mathrm{P})$ with the same boundary condition $x$ and with different large enough real numbers $T$. This information can be useful if we need to find an approximate solution of the problem $(\mathrm{P})$ with the boundary condition $x$ and with a new interval $[0, T]$ where $T$ is large enough. This approximate solution is the concatenation of the approximation of $Y^{(f, x)}(t)$, the turnpike $\bar{x}$ and the approximation of $\Lambda^{\left(x_{*}\right)}(T-t)$.

\section{Auxiliary Results for the Proof of Theorem 3.2}

Proposition 4.1 ([2], Chap. 10, [19], Prop. 2.2). Let $T>0$ and let $v_{k}:[0, T] \rightarrow R^{n}, k=1,2, \ldots$ be a sequence of a.c. functions such that the sequence $\left\{I^{f}\left(0, T, v_{k}\right)\right\}_{k=1}^{\infty}$ is bounded and that the sequence $\left\{v_{k}(0)\right\}_{k=1}^{\infty}$ is bounded. Then there exist a strictly increasing sequence of natural numbers $\left\{k_{i}\right\}_{i=1}^{\infty}$ and an a.c. function $v:[0, T] \rightarrow R^{n}$ such that

$$
\begin{gathered}
v_{k_{i}}(t) \rightarrow v(t) \text { as } i \rightarrow \infty \text { uniformly on }[0, T], \\
I^{f}(0, T, v) \leq \liminf _{i \rightarrow \infty} I^{f}\left(0, T, v_{k_{i}}\right) .
\end{gathered}
$$

Proposition 4.2 ([19], Prop. 2.1). Let $M_{0}, M_{1}$ be positive numbers. Then there exists $M_{2}>0$ such that for each $T>0$ and each a.c. function $v:[0, T] \rightarrow R^{n}$ which satisfies

$$
|v(0)| \leq M_{0}, I^{f}(0, T, v) \leq T f(\bar{x}, 0)+M_{1}
$$

the following inequality holds:

$$
|v(t)| \leq M_{2} \text { for all } t \in[0, T] .
$$

Proposition 4.3 ([19], Prop. 2.3). Let $\epsilon>0$. Then there exists $\delta>0$ such that if an a.c. function $v:[0,1] \rightarrow R^{n}$ satisfies $|v(0)-\bar{x}|,|v(1)-\bar{x}| \leq \delta$, then

$$
I^{f}(0,1, v) \geq f(\bar{x}, 0)-\epsilon .
$$

\section{Proof of Theorem 3.2}

For simplicity we use the notation $Y^{(z)}=Y^{(f, z)}$ for each $z \in \cup\left\{X_{M}: M \in(0, \infty)\right\}$.

Assume that the assertion of the theorem does not hold. Therefore for each integer $k$ there exists

$$
T_{k} \geq L_{0}+4 k
$$

and an a.c. function $v_{k}:\left[0, T_{k}\right] \rightarrow R^{n}$ such that

$$
\begin{gathered}
v_{k}(0) \in X_{M}, I^{f}\left(0, T_{k}, v_{k}\right) \leq \sigma\left(f, T_{k}, v_{k}(0)\right)+k^{-1}, \\
\sup \left\{\left|v_{k}(t)-Y^{\left(v_{k}(0)\right)}(t)\right|: t \in\left[0, L_{0}\right]\right\}>\epsilon .
\end{gathered}
$$

In the first step of the proof we obtain some useful estimates for $\left|v_{k}(t)\right|, t \in\left[0, T_{k}\right]$ and $\left|Y^{\left(v_{k}(0)\right)}(t)\right|, t \in[0, \infty)$ and for the integral functional with the integrand $f$ and the functions $\left|v_{k}\right|$ and $\left|Y^{\left(v_{k}(0)\right)}\right|, k=1,2, \ldots$

It follows from (5.2) and the definition of $X_{M}$ (see (2.9)) that for each integer $k \geq 1$

$$
\begin{gathered}
I^{f}\left(0, T_{k}, v_{k}\right) \leq \sigma\left(f, T_{k}, v_{k}(0)\right)+k^{-1} \leq M+T_{k} f(\bar{x}, 0)+k^{-1}, \\
\left|v_{k}(0)\right| \leq M .
\end{gathered}
$$


By (5.4), (5.5) and Proposition 4.2 there exists $M_{0}>0$ such that for each integer $k \geq 1$

$$
\left|v_{k}(t)\right| \leq M_{0} \text { for all } t \in\left[0, T_{k}\right] .
$$

In view of (5.2) and the definition of $X_{M}$ (see (2.9)) for each natural number $k$

$$
I^{f}\left(0, T, Y^{\left(v_{k}(0)\right)}\right) \leq T f(\bar{x}, 0)+M+1 \text { for all large enough } T .
$$

Together with Proposition 4.2 and (5.5) this implies that there exists $M_{1}>M_{0}$ such that for each natural number $k$

$$
\left|Y^{\left(v_{k}(0)\right)}(t)\right| \leq M_{1} \text { for all } t \in[0, \infty) .
$$

By Proposition 2.1, there exists $c_{1}>0$ such that

$$
\sigma(f, T, x) \geq T f(\bar{x}, 0)-c_{1} \text { for each } T>0 \text { and each } x \in R^{n} \text { satisfying }|x| \leq M_{1} .
$$

In our second step of the proof we show the existence of a subsequence $\left\{v_{k_{i}}\right\}_{i=1}^{\infty}$ and an interval $\left[a_{0}, b_{0}\right] \subset$ $\left(0, L_{0}\right]$ such that $\left|v_{k_{i}}(t)-Y^{\left(v_{k_{i}}(0)\right)}(t)\right| \geq \epsilon / 4$ for all $t \in\left[a_{0}, b_{0}\right]$ and all large enough integers $i$. Moreover, we show that $v_{k_{i}}$ (respectively, $Y^{\left(v_{k_{i}}(0)\right)}$ ) converges to $\tilde{v}$ (respectively, $\tilde{y}$ ) as $i \rightarrow \infty$ uniformly on any bounded subinterval of $[0, \infty)$.

Fix an integer $j \geq 1$. In view of (5.1), (5.4), (5.6), (5.9) and the relation $M_{1}>M_{0}$ for each integer $k \geq j$

$$
\begin{aligned}
I^{f}\left(0, j, v_{k}\right)= & I^{f}\left(0, T_{k}, v_{k}\right)-I^{f}\left(j, T_{k}, v_{k}\right) \leq M+k^{-1}+T_{k} f(\bar{x}, 0)-\sigma\left(f, T_{k}-j, v_{k}(j)\right) \\
& \leq M+k^{-1}+T_{k} f(\bar{x}, 0)-\left(T_{k}-j\right) f(\bar{x}, 0)+c_{1} \leq M+k^{-1}+j f(\bar{x}, 0)+c_{1} .
\end{aligned}
$$

Let $k$ be a natural number. In view of (5.7) there is $S_{k}>2 j+2$ such that

$$
I^{f}\left(0, S_{k}, Y^{\left(v_{k}(0)\right)}\right) \leq S_{k} f(\bar{x}, 0)+M+1 .
$$

It follows from (5.11), (5.8) and (5.9) that

$$
\begin{aligned}
I^{f}\left(0, j, Y^{\left(v_{k}(0)\right)}\right) & =I^{f}\left(0, S_{k}, Y^{\left(v_{k}(0)\right)}\right)-I^{f}\left(j, S_{k}, Y^{\left(v_{k}(0)\right)}\right) \\
& \leq S_{k} f(\bar{x}, 0)+M+1-\sigma\left(f, S_{k}-j, Y^{\left(v_{k}(0)\right)}(j)\right) \\
& \leq S_{k} f(\bar{x}, 0)+M+1-\left(S_{k}-j\right) f(\bar{x}, 0)+c_{1}=j f(\bar{x}, 0)+M+1+c_{1} .
\end{aligned}
$$

By Proposition 4.1, (5.11), (5.12) and (5.5) extracting a subsequence and re-indexing we may assume without loss of generality that there exist a strictly increasing sequence of natural numbers $\left\{k_{i}\right\}_{i=1}^{\infty}$ and a.c. functions $\tilde{v}:[0, \infty) \rightarrow R^{n}$ and $\tilde{y}:[0, \infty) \rightarrow R^{n}$ such that for each integer $j \geq 1$

$$
\begin{gathered}
v_{k_{i}}(t) \rightarrow \tilde{v}(t) \text { as } i \rightarrow \infty \text { uniformly on }[0, j], \\
Y^{\left(v_{k_{i}}(0)\right)}(t) \rightarrow \tilde{y}(t) \text { as } i \rightarrow \infty \text { uniformly on }[0, j], \\
I^{f}(0, j, \tilde{v}) \leq \liminf _{i \rightarrow \infty} I^{f}\left(0, j, v_{k_{i}}\right), \\
I^{f}(0, j, \tilde{y}) \leq \liminf _{i \rightarrow \infty} I^{f}\left(0, j, Y^{\left(v_{k_{i}}(0)\right)}\right) .
\end{gathered}
$$

Relations (5.10), (5.12) and (5.13) imply that for each integer $j \geq 1$

$$
I^{f}(0, j, \tilde{v}) \leq M+j f(\bar{x}, 0)+c_{1}, I^{f}(0, j, \tilde{y}) \leq j f(\bar{x}, 0)+M+1+c_{1} .
$$


In view of (5.14) and Proposition 2.2, $\tilde{v}$ and $\tilde{y}$ are $(f)$-good functions. Combined with Proposition 2.3 this implies that

$$
\tilde{v}(t) \rightarrow \bar{x}, \tilde{y}(t) \rightarrow \bar{x} \text { as } t \rightarrow \infty .
$$

By (5.3) and (5.13),

$$
\sup \left\{|\tilde{v}(t)-\tilde{y}(t)|: t \in\left[0, L_{0}\right]\right\} \geq \epsilon / 2 .
$$

Relation (5.16) implies that there exist $a_{0}, b_{0} \in\left(0, L_{0}\right]$ such that

$$
0<b_{0}-a_{0}<1 \text { and }|\tilde{v}(t)-\tilde{y}(t)| \geq \epsilon / 3 \text { for all } t \in\left[a_{0}, b_{0}\right] .
$$

In view of (5.13) and (5.17) there is an integer $i_{0} \geq 4+L_{0}$ such that for each integer $i \geq i_{0}$,

$$
\left|v_{k_{i}}(t)-Y^{\left(v_{k_{i}}(0)\right)}(t)\right| \geq \epsilon / 4 \text { for all } t \in\left[a_{0}, b_{0}\right] .
$$

In our third step we show that the values of the integral functional with the integrand $f$ and with the functions $\left|v_{k_{i}}\right|$ and $\left|Y^{\left(v_{k_{i}}(0)\right)}\right|, i=1,2, \ldots$ are bounded by a constant which does not depend on $i$.

Assume that an integer $i$ satisfies $i \geq i_{0}$. By (5.5), (5.6), (5.9) and the relation $M_{1}>M_{0}$

$$
\begin{aligned}
I^{f}\left(a_{0}, b_{0}, v_{k_{i}}\right) & =I^{f}\left(0, T_{k_{i}}, v_{k_{i}}\right)-I^{f}\left(0, a_{0}, v_{k_{i}}\right)-I^{f}\left(b_{0}, T_{k_{i}}, v_{k_{i}}\right) \\
& \leq M+T_{k_{i}} f(\bar{x}, 0)+1-\sigma\left(f, a_{0}, v_{k_{i}}(0)\right)-\sigma\left(f, T_{k_{i}}-b_{0}, v_{k_{i}}\left(b_{0}\right)\right) \\
& \leq M+T_{k_{i}} f(\bar{x}, 0)+1-a_{0} f(\bar{x}, 0)+c_{1}-\left(T_{k_{i}}-b_{0}\right) f(\bar{x}, 0)+c_{1} \\
& \leq M+\left(b_{0}-a_{0}\right) f(\bar{x}, 0)+2 c_{1}+1 .
\end{aligned}
$$

In view of (5.7) there is $S_{i}>4 b_{0}+4$ such that

$$
I^{f}\left(0, S_{i}, Y^{\left(v_{k_{i}}(0)\right)}\right) \leq S_{i} f(\bar{x}, 0)+M+1 .
$$

It follows from (5.8), (5.9) and (5.20) that

$$
\begin{aligned}
I^{f}\left(a_{0}, b_{0}, Y^{\left(v_{k_{i}}(0)\right)}\right) & =I^{f}\left(0, S_{i}, Y^{\left(v_{k_{i}}(0)\right)}\right)-I^{f}\left(0, a_{0}, Y^{\left(v_{k_{i}}(0)\right)}\right)-I^{f}\left(b_{0}, S_{i}, Y^{\left(v_{k_{i}}(0)\right)}\right) \\
& \leq S_{i} f(\bar{x}, 0)+M+1-\sigma\left(f, a_{0}, Y^{\left(v_{k_{i}}(0)\right)}(0)\right)-\sigma\left(f, S_{i}-b_{0}, Y^{\left(v_{k_{i}}(0)\right)}\left(b_{0}\right)\right) \\
& \leq S_{i} f(\bar{x}, 0)+M+1-a_{0} f(\bar{x}, 0)+c_{1}-\left(S_{i}-b_{0}\right) f(\bar{x}, 0)+c_{1} \\
& =\left(b_{0}-a_{0}\right) f(\bar{x}, 0)+2 c_{1}+M+1 .
\end{aligned}
$$

By (5.19) and (5.20) for each integer $i \geq i_{0}$,

$$
\begin{aligned}
& I^{f}\left(a_{0}, b_{0}, v_{k_{i}}\right), I^{f}\left(a_{0}, b_{0}, Y^{\left(v_{k_{i}}(0)\right)}\right) \\
& \leq M+2 c_{1}+1+\left(b_{0}-a_{0}\right) f(\bar{x}, 0) .
\end{aligned}
$$

In the fourth step of the proof we show that there is a constant $\gamma_{0}>0$ such that for each integer $i \geq i_{0}$ and each $S \in\left[L_{0}, T_{k_{i}}\right]$,

$$
I^{f}\left(0, S, 2^{-1}\left(v_{k_{i}}+Y^{\left(v_{k_{i}}(0)\right)}\right) \leq 2^{-1} I^{f}\left(0, S, v_{k_{i}}\right)+2^{-1} I^{f}\left(0, S, Y^{\left(v_{k_{i}}(0)\right)}\right)-\gamma_{0}(3 / 4)\left(b_{0}-a_{0}\right) .\right.
$$

In view of (2.1) there exists a number $M_{2}>M_{1}+1$ such that

$$
\psi\left(M_{2}\right)>4\left[2 a+2\left(M+2 c_{1}+1\right)\left(b_{0}-a_{0}\right)^{-1}+|f(\bar{x}, 0)|\right] .
$$


For each integer $i \geq i_{0}$ set

$$
\begin{gathered}
E_{i}=\left\{t \in\left[a_{0}, b_{0}\right]:\left|v_{k_{i}}^{\prime}(t)\right|,\left|\left(Y^{\left(v_{k_{i}}(0)\right)}\right)^{\prime}(t)\right| \leq M_{2}\right\} \\
\cap\left\{t \in\left[a_{0}, b_{0}\right]: f\left(v_{k_{i}}(t), v_{k_{i}}^{\prime}(t)\right), f\left(Y^{\left(v_{k_{i}}(0)\right)}(t),\left(Y^{\left(v_{k_{i}}(0)\right)}\right)^{\prime}(t)\right)<\infty\right\} .
\end{gathered}
$$

Assume that an integer $i \geq i_{0}$. It follows from (5.22), (2.2), (5.24) and the monotonicity of $\psi$ that

$$
\begin{aligned}
2\left(M+2 c_{1}+1+\right. & \left.\left(b_{0}-a_{0}\right) f(\bar{x}, 0)\right) \geq I^{f}\left(a_{0}, b_{0}, v_{k_{i}}\right)+I^{f}\left(a_{0}, b_{0}, Y^{\left(v_{k_{i}}(0)\right)}\right) \\
& \geq \int_{a_{0}}^{b_{0}}\left(\psi\left(\left|v_{k_{i}}^{\prime}(t)\right|\right)\left|v_{i_{k}}^{\prime}(t)\right|-a\right) \mathrm{d} t+\int_{a_{0}}^{b_{0}}\left(\psi\left(\left|\left(Y^{\left(v_{k_{i}}(0)\right)}\right)^{\prime}(t)\right|\right)\left|\left(Y^{\left(v_{i_{k}}(0)\right)}\right)^{\prime}(t)\right|-a\right) \mathrm{d} t \\
& \geq-2\left(b_{0}-a_{0}\right) a+\operatorname{mes}\left(\left[a_{0}, b_{0}\right] \backslash E_{i}\right) \psi\left(M_{2}\right) M_{2} .
\end{aligned}
$$

Together with (5.23) and the inequality $M_{2}>M_{1}+1$ this relation implies that

$$
\begin{aligned}
\operatorname{mes}\left(\left[a_{0}, b_{0}\right] \backslash E_{i}\right) & \leq\left(\psi\left(M_{2}\right) M_{2}\right)^{-1}\left[2\left(b_{0}-a_{0}\right) a+2\left(M+2 c_{1}+1+\left(b_{0}-a_{0}\right)|f(\bar{x}, 0)|\right)\right] \\
& \leq\left(\psi\left(M_{2}\right)\right)^{-1}\left[2\left(b_{0}-a_{0}\right) a+2\left(M+2 c_{1}+1+\left(b_{0}-a_{0}\right)|f(\bar{x}, 0)|\right] \leq\left(b_{0}-a_{0}\right) / 4\right.
\end{aligned}
$$

Relations (5.24) and (5.26) imply that

$$
\operatorname{mes}\left(E_{i}\right) \geq(3 / 4)\left(b_{0}-a_{0}\right) \text { for each integer } i \geq i_{0} .
$$

By (A2) there exists $\gamma_{0} \in(0,1)$ such that for each $\left(\xi_{1}, \xi_{2}\right),\left(\eta_{1}, \eta_{2}\right) \in \operatorname{dom}(f)$ which satisfy

$$
\left|\xi_{i}\right|,\left|\eta_{i}\right| \leq M_{2}, i=1,2,\left|\xi_{1}-\xi_{2}\right| \geq \epsilon / 8
$$

the following inequality holds:

$$
-f\left(2^{-1}\left(\xi_{1}+\xi_{2}\right), 2^{-1}\left(\eta_{1}+\eta_{2}\right)\right)+2^{-1} f\left(\xi_{1}, \eta_{1}\right)+2^{-1} f\left(\xi_{2}, \eta_{2}\right) \geq \gamma_{0} .
$$

For each integer $i \geq i_{0}$ define

$$
u_{i}(t)=2^{-1}\left(v_{k_{i}}(t)+Y^{\left(v_{k_{i}}(0)\right)}(t)\right), t \in\left[0, T_{k_{i}}\right] .
$$

Let an integer $i \geq i_{0}$. By (5.30) for almost every $t \in\left[0, T_{k_{i}}\right]$,

$$
f\left(u_{i}(t), u_{i}^{\prime}(t)\right) \leq 2^{-1} f\left(v_{k_{i}}(t), v_{k_{i}}^{\prime}(t)\right)+2^{-1} f\left(Y^{\left(v_{k_{i}}(0)\right)}(t),\left(Y^{\left(v_{k_{i}}(0)\right)}\right)^{\prime}(t)\right) .
$$

In view of (5.24), the relation $M_{2}>M_{1}+1>M_{0},(5.8),(5.6),(5.18)$, the choice of $\gamma_{0}$ (see (5.28) and (5.29)) and (5.30) for almost every $t \in E_{i}$,

$$
f\left(u_{i}(t), u_{i}^{\prime}(t)\right) \leq 2^{-1} f\left(v_{i_{k}}(t), v_{k_{i}}^{\prime}(t)\right)+2^{-1} f\left(Y^{\left(v_{k_{i}}(0)\right)}(t),\left(Y^{\left(v_{k_{i}}(0)\right)}\right)^{\prime}(t)\right)-\gamma_{0} .
$$

It follows from $(5.24),(5.27),(5.31),(5.32)$ and the inclusions $a_{0}, b_{0} \in\left[0, L_{0}\right]$ that for each $S \in\left[L_{0}, T_{k_{i}}\right]$,

$$
\begin{aligned}
I^{f}\left(0, S, u_{i}\right) & \leq 2^{-1} I^{f}\left(0, S, v_{k_{i}}\right)+2^{-1} I^{f}\left(0, S, Y^{\left(v_{k_{i}}(0)\right)}\right)-\gamma_{0} \operatorname{mes}\left(E_{i}\right) \\
& \leq 2^{-1} I^{f}\left(0, S, v_{k_{i}}\right)+2^{-1} I^{f}\left(0, S, Y^{\left(v_{k_{i}}(0)\right)}\right)-\gamma_{0}(3 / 4)\left(b_{0}-a_{0}\right) .
\end{aligned}
$$

Now we turn to the fifth step of our proof. Here we first need to choose certain constants.

Set

$$
\Delta=\gamma_{0}\left(b_{0}-a_{0}\right) / 16
$$


By (A1), the continuity of $f$ at $(\bar{x}, 0)$ and Proposition 4.3 there exists $r \in(0,1)$ such that:

$$
\begin{gathered}
\left\{\left(\xi_{1}, \xi_{2}\right) \in R^{n} \times R^{n}:\left|\xi_{1}-\bar{x}\right| \leq 4 r \text { and }\left|\xi_{2}\right| \leq 4 r\right\} \subset \operatorname{dom}(f) ; \\
\qquad\left|f\left(\xi_{1}, \xi_{2}\right)-f(\bar{x}, 0)\right| \leq 32^{-1} \Delta \\
\text { for each } \xi_{1}, \xi_{2} \in R^{n} \text { satisfying }\left|\xi_{1}-\bar{x}\right| \leq 4 r,\left|\xi_{2}\right| \leq 4 r ;
\end{gathered}
$$

if an a.c. function $h:[0,1] \rightarrow R^{n}$ satisfies $|h(0)-\bar{x}|,|h(1)-\bar{x}| \leq 4 r$, then

$$
I^{f}(0,1, h) \geq f(\bar{x}, 0)-\Delta / 16
$$

In view of (5.15) there exists a natural number $L_{2}$ such that

$$
|\tilde{v}(t)-\bar{x}|,|\tilde{y}(t)-\bar{x}| \leq r / 8 \text { for all } t \geq L_{2} .
$$

By (5.13) there exists an integer $j \geq i_{0}+4 L_{2}+4$ such that

$$
\begin{gathered}
k_{j}^{-1}<16^{-1} \Delta \\
\left|v_{k_{j}}(t)-\tilde{v}(t)\right|,\left|Y^{\left(v_{k_{j}}(0)\right)}(t)-\tilde{y}(t)\right| \leq r / 32 \text { for all } t \in\left[0,4 L_{2}+4 L_{0}+4\right] .
\end{gathered}
$$

We consider the function $u_{j}$ defined by $(5.30)$ and define a.c. functions $u_{j}^{(1)}, u_{j}^{(2)}:\left[0,4 L_{0}+4 L_{2}+4\right] \rightarrow R^{n}$ as follows:

$$
\begin{gathered}
u_{j}^{(1)}(t)=u_{j}(t), t \in\left[0,4 L_{0}+4 L_{2}+3\right], \\
u_{j}^{(1)}(t)=u_{j}\left(4 L_{0}+4 L_{2}+3\right) \\
+\left(t-\left(4 L_{0}+4 L_{2}+3\right)\right)\left[v_{k_{j}}\left(4 L_{0}+4 L_{2}+4\right)-u_{j}\left(4 L_{0}+4 L_{2}+3\right)\right], \\
t \in\left[4 L_{0}+4 L_{2}+3,4 L_{0}+4 L_{2}+4\right], \\
u_{j}^{(2)}(t)=u_{j}(t), t \in\left[0,4 L_{0}+4 L_{2}+3\right], \\
u_{j}^{(2)}(t)=\left(t-\left(4 L_{0}+4 L_{2}+3\right)\right)\left[Y^{\left(v_{k_{j}}(0)\right)}\left(4 L_{0}+4 L_{2}+4\right)-u_{j}\left(4 L_{0}+4 L_{2}+3\right)\right] \\
+u_{j}\left(4 L_{0}+4 L_{2}+3\right), t \in\left[4 L_{0}+4 L_{2}+3,4 L_{0}+4 L_{2}+4\right] .
\end{gathered}
$$

It is clear that

$$
\begin{gathered}
u_{j}^{(2)}(0)=u_{j}^{(1)}(0)=u_{j}(0)=v_{k_{j}}(0)=Y^{\left(v_{k_{j}}(0)\right)}(0), \\
u_{j}^{(1)}\left(4 L_{0}+4 L_{2}+4\right)=v_{k_{j}}\left(4 L_{0}+4 L_{2}+4\right), \\
u_{j}^{(2)}\left(4 L_{0}+4 L_{2}+4\right)=Y^{\left(v_{k_{j}}(0)\right)}\left(4 L_{0}+4 L_{2}+4\right) .
\end{gathered}
$$

Since the function $Y^{\left(v_{k_{j}}(0)\right)}$ is $(f)$-overtaking optimal (5.43) implies that

$$
I^{f}\left(0,4 L_{0}+4 L_{2}+4, u_{j}^{(2)}\right) \geq I^{f}\left(0,4 L_{0}+4 L_{2}+4, Y^{\left(v_{k_{j}}(0)\right)}\right) .
$$

In view of (5.2) and (5.43)

$$
I^{f}\left(0,4 L_{0}+4 L_{2}+4, u_{j}^{(1)}\right) \geq I^{f}\left(0,4 L_{0}+4 L_{2}+4, v_{k_{j}}\right)-k_{j}^{-1} .
$$

Relations (5.38) and (5.40) imply that for all $t \in\left[L_{2}, 4 L_{2}+4 L_{0}+4\right]$,

$$
\left|v_{k_{j}}(t)-\bar{x}\right| \leq r / 32+r / 8,\left|Y^{\left(v_{k_{j}}(0)\right)}(t)-\bar{x}\right| \leq r / 32+r / 8 .
$$


By (5.46) and the choice of $r$ (see (5.37))

$$
\begin{gathered}
I^{f}\left(4 L_{2}+4 L_{0}+3,4 L_{2}+4 L_{0}+4, v_{k_{j}}\right) \geq f(\bar{x}, 0)-\Delta / 16, \\
I^{f}\left(4 L_{2}+4 L_{0}+3,4 L_{2}+4 L_{0}+4, Y^{\left(v_{k_{j}}(0)\right)}\right) \geq f(\bar{x}, 0)-\Delta / 16 .
\end{gathered}
$$

Let $t \in\left[4 L_{2}+4 L_{0}+3,4 L_{2}+4 L_{0}+4\right]$. We evaluate $f\left(u_{j}^{(p)}(t),\left(u_{j}^{(p)}\right)^{\prime}(t)\right)$ for $p=1,2$. Relations (5.30), (5.41) and (5.46) imply that

$$
\begin{aligned}
\left|u_{j}^{(1)}(t)-\bar{x}\right| & \leq \max \left\{\left|u_{j}\left(4 L_{0}+4 L_{2}+3\right)-\bar{x}\right|,\left|v_{k_{j}}\left(4 L_{0}+4 L_{2}+4\right)-\bar{x}\right|\right\} \\
& \leq \max \left\{\left|v_{k_{j}}\left(4 L_{0}+4 L_{2}+3\right)-\bar{x}\right|,\left|Y^{\left(v_{k_{j}}(0)\right)}\left(4 L_{0}+4 L_{2}+3\right)-\bar{x}\right|,\left|v_{k_{j}}\left(4 L_{0}+4 L_{2}+4\right)-\bar{x}\right|\right\} \\
& \leq r / 32+r / 8 .
\end{aligned}
$$

It follows from (5.41) and (5.46) that

$$
\begin{aligned}
\left|\left(u_{j}^{(1)}\right)^{\prime}(t)\right| & \leq\left|v_{k_{j}}\left(4 L_{0}+4 L_{2}+4\right)-u_{j}\left(4 L_{0}+4 L_{0}+3\right)\right| \\
& \leq\left|v_{k_{j}}\left(4 L_{0}+4 L_{2}+4\right)-\bar{x}\right|+\left|\bar{x}-u_{j}\left(4 L_{0}+4 L_{2}+3\right)\right| \leq 2(r / 32+r / 8) .
\end{aligned}
$$

By (5.30), (5.42) and (5.46)

$$
\begin{aligned}
\left|u_{j}^{(2)}(t)-\bar{x}\right| \leq & \max \left\{\left|Y^{\left(v_{k_{j}}(0)\right)}\left(4 L_{0}+4 L_{2}+4\right)-\bar{x}\right|,\left|u_{j}\left(4 L_{0}+4 L_{2}+3\right)-\bar{x}\right|\right\} \\
\leq & \max \left\{\left|Y^{\left(v_{k_{j}}(0)\right)}\left(4 L_{0}+4 L_{2}+4\right)-\bar{x}\right|,\left|Y^{\left(v_{k_{j}}(0)\right)}\left(4 L_{0}+4 L_{2}+3\right)-\bar{x}\right|,\right. \\
& \left.\left|v_{k_{j}}\left(4 L_{0}+4 L_{2}+3\right)-\bar{x}\right|\right\} \leq r / 32+r / 8 .
\end{aligned}
$$

In view of (5.42) and (5.46)

$$
\begin{aligned}
\left|\left(u_{j}^{(2)}\right)^{\prime}(t)\right| & \leq\left|Y^{\left(v_{k_{j}}(0)\right)}\left(4 L_{0}+4 L_{2}+4\right)-u_{j}\left(4 L_{0}+4 L_{2}+3\right)\right| \\
& \leq\left|Y^{\left(v_{k_{j}}(0)\right)}\left(4 L_{0}+4 L_{2}+4\right)-\bar{x}\right|+\left|\bar{x}-u_{j}\left(4 L_{0}+4 L_{2}+3\right)\right| \leq 2(r / 32+r / 8) .
\end{aligned}
$$

Relations (5.36), (5.48)-(5.51) imply that

$$
\begin{gathered}
\left|f\left(u_{j}^{(p)}(t),\left(u_{j}^{(p)}\right)^{\prime}(t)\right)-f(\bar{x}, 0)\right| \leq 32^{-1} \Delta \\
\text { for } p=1,2 \text { and all } t \in\left[4 L_{2}+4 L_{0}+3,4 L_{2}+4 L_{0}+4\right] .
\end{gathered}
$$

It follows from (5.41), (5.42), (5.52), (5.45), (5.44), (5.39) and (5.47) that

$$
\begin{aligned}
2 I^{f}\left(0,4 L_{0}+4 L_{2}+3, u_{j}\right)= & I^{f}\left(0,4 L_{0}+4 L_{2}+4, u_{j}^{(1)}\right)+I^{f}\left(0,4 L_{0}+4 L_{2}+4, u_{j}^{(2)}\right) \\
& -I^{f}\left(4 L_{0}+4 L_{2}+3,4 L_{0}+4 L_{2}+4, u_{j}^{(1)}\right)-I^{f}\left(4 L_{0}+4 L_{2}+3,4 L_{0}+4 L_{2}+4, u_{j}^{(2)}\right) \\
\geq & I^{f}\left(0,4 L_{0}+4 L_{2}+4, u_{j}^{(1)}\right)+I^{f}\left(0,4 L_{0}+4 L_{2}+4, u_{j}^{(2)}\right)-16^{-1} \Delta-2 f(\bar{x}, 0) \\
\geq & I^{f}\left(0,4 L_{0}+4 L_{2}+4, v_{k_{j}}\right)-k_{j}^{-1}+I^{f}\left(0,4 L_{0}+4 L_{2}+4, Y^{\left(v_{k_{j}}(0)\right)}\right)-16^{-1} \Delta-2 f(\bar{x}, 0) \\
\geq & I^{f}\left(0,4 L_{0}+4 L_{2}+4, v_{k_{j}}\right)+I^{f}\left(0,4 L_{0}+4 L_{2}+4, Y^{\left(v_{k_{j}}(0)\right)}\right)-8^{-1} \Delta-2 f(\bar{x}, 0) \\
\geq & I^{f}\left(0,4 L_{0}+4 L_{2}+3, v_{k_{j}}\right)+I^{f}\left(0,4 L_{0}+4 L_{2}+3, Y^{\left(v_{k_{j}}(0)\right)}\right)+2 f(\bar{x}, 0)-\Delta / 4-2 f(\bar{x}, 0) \\
\geq & 2 I^{f}\left(0,4 L_{0}+4 L_{2}+3, u_{j}\right)+\gamma_{0}\left(b_{0}-a_{0}\right)-\Delta / 4 \\
\geq & 2 I^{f}\left(0,4 L_{0}+4 L_{2}+3, u_{j}\right)+\gamma_{0}\left(b_{0}-a_{0}\right) / 2 .
\end{aligned}
$$

The contradiction we have reached proves Theorem 3.2. 


\section{Proof of Proposition 3.1}

By (3.3) and (3.5) there exists $a_{0}>a$ such that for each $x, y \in R^{n}$,

$$
\bar{L}(x, y) \geq(3 / 4) \max \{\psi(|x|), \psi(|y|)|y|\}-a_{0}
$$

Lemma 6.1. Let $g \in\{\bar{L}, \bar{f}\}$ and let $M>0$. Then there exists $M_{0}>0$ such that for each a.c. function $v:[0,1] \rightarrow R^{n}$ satisfying $\int_{0}^{1} g\left(v(t), v^{\prime}(t)\right) \mathrm{d} t \leq M$ the inequality $|v(t)| \leq M_{0}$ holds for all $t \in[0,1]$.

Proof. In view of (2.1) there exists $M_{1}>0$ such that

$$
\psi\left(M_{1}\right)>\left(M+a_{0}\right) 4
$$

and there is $a_{1}>0$ such that

$$
4^{-1} \psi(t) t \geq t-a_{1} \text { for all } t \geq 0
$$

Choose a number

$$
M_{0}>a_{0}+a_{1}+M+M_{1}
$$

Assume that an a.c. function $v:[0,1] \rightarrow R^{n}$ satisfies

$$
\int_{0}^{1} g\left(v(t), v^{\prime}(t)\right) \mathrm{d} t \leq M
$$

We will show that

$$
|v(t)| \leq M_{0} \text { for all } t \in[0,1] .
$$

Let us assume the contrary. Then there exists $t_{0} \in[0,1]$ such that

$$
\left|v\left(t_{0}\right)\right|>M_{0}
$$

If $|v(t)| \geq M_{1}$ for all $\in[0,1]$, then relations (3.3), (6.1) and (6.2) imply that

$$
M \geq \int_{0}^{1} g\left(v(t), v^{\prime}(t)\right) \mathrm{d} t \geq(3 / 4) \psi\left(M_{1}\right)-a_{0} .
$$

This contradicts (6.2). The contradiction we have reached proves that there is $t_{1} \in[0,1]$ such that

$$
\left|v\left(t_{1}\right)\right|<M_{1}
$$

Clearly, $t_{1} \neq t_{0}$. By (6.6), the inequality $\left|v\left(t_{1}\right)\right|<M_{1},(3.3),(6.1),(6.3)$ and (6.5),

$$
\begin{aligned}
M_{0}-M_{1} & \leq\left|v\left(t_{0}\right)\right|-\left|v\left(t_{1}\right)\right| \leq\left|\int_{t_{1}}^{t_{0}}\right| v^{\prime}(t)|\mathrm{d} t| \leq\left|\int_{t_{1}}^{t_{0}}\left[a_{1}+4^{-1} \psi\left(\left|v^{\prime}(t)\right|\right)\left|v^{\prime}(t)\right|\right] \mathrm{d} t\right| \\
& \leq a_{1}+\left|\int_{t_{1}}^{t_{0}}\left[g\left(v(t), v^{\prime}(t)\right)+a_{0}\right] \mathrm{d} t\right| \leq a_{1}+a_{0}+\int_{0}^{1} g\left(v(t), v^{\prime}(t)\right) \mathrm{d} t \leq a_{0}+a_{1}+M .
\end{aligned}
$$

This contradicts (6.4). The contradiction we have reached proves Lemma 6.1.

Proposition 6.1. An a.c. function $v:[0, \infty) \rightarrow R^{n}$ is $(\bar{f})$-good if and only if

$$
\int_{0}^{\infty} \bar{L}\left(v(t), v^{\prime}(t)\right) \mathrm{d} t:=\lim _{T \rightarrow \infty} \int_{0}^{T} \bar{L}\left(v(t), v^{\prime}(t)\right) \mathrm{d} t<\infty .
$$


Proof. Let $v:[0, \infty) \rightarrow R^{n}$ be an a.c. function. Then in view of (3.5) for each $T>0$,

$$
\begin{aligned}
\int_{0}^{T} \bar{f}\left(v(t), v^{\prime}(t)\right) \mathrm{d} t & =\int_{0}^{T} \bar{L}\left(v(t), v^{\prime}(t)\right) \mathrm{d} t+T \bar{f}(\bar{x}, 0)-\int_{0}^{T}\left\langle l, v^{\prime}(t)\right\rangle \mathrm{d} t \\
& =\int_{0}^{T} \bar{L}\left(v(t), v^{\prime}(t)\right) \mathrm{d} t+T \bar{f}(\bar{x}, 0)-\langle l, v(T)-v(0)\rangle .
\end{aligned}
$$

If $v$ is $(\bar{f})$-good, then by Proposition 2.2 the function $v$ is bounded and

$$
\int_{0}^{\infty} \bar{L}\left(v(t), v^{\prime}(t)\right) \mathrm{d} t<\infty .
$$

If (6.8) holds, then by Lemma 6.1 the function $v$ is bounded on $[0, \infty)$ and (6.7) and Proposition 2.2 imply that $v$ is $(\bar{f})$-good. Proposition 6.1 is proved.

Proposition 6.2. Let $x \in \bar{X}$ and let an $(\bar{f})$-good function $v:[0, \infty) \rightarrow R^{n}$ satisfies $v(0)=x$. Then

$$
\int_{0}^{\infty} \bar{L}\left(v(t), v^{\prime}(t)\right) \mathrm{d} t-\langle l, \bar{x}-x\rangle \geq \pi(x) .
$$

Proof. Since the function $\Lambda^{(x)}$ is $(\bar{f})$-overtaking optimal it is also $(\bar{f})$-good and in view of (3.5), Propositions 6.1 and 2.3 ,

$$
\begin{aligned}
0 & \leq \limsup _{T \rightarrow \infty}\left[\int_{0}^{T} \bar{f}\left(\Lambda^{(x)}(t),\left(\Lambda^{(x)}\right)^{\prime}(t)\right) \mathrm{d} t-\int_{0}^{T} \bar{f}\left(v(t), v^{\prime}(t)\right) \mathrm{d} t\right] \\
& =\limsup _{T \rightarrow \infty}\left[\int_{0}^{T} \bar{L}\left(\Lambda^{(x)}(t),\left(\Lambda^{(x)}\right)^{\prime}(t)\right) \mathrm{d} t-\int_{0}^{T} \bar{L}\left(v(t), v^{\prime}(t)\right) \mathrm{d} t-\left\langle l,-x+\Lambda^{(x)}(T)\right\rangle+\langle l, v(T)-x\rangle\right] \\
& =\int_{0}^{\infty} \bar{L}\left(\Lambda^{(x)}(t),\left(\Lambda^{(x)}\right)^{\prime}(t)\right) \mathrm{d} t-\langle l, \bar{x}-x\rangle-\int_{0}^{\infty} \bar{L}\left(v(t), v^{\prime}(t)\right) \mathrm{d} t+\langle l, \bar{x}-x\rangle .
\end{aligned}
$$

Together with (3.11) this implies that

$$
\int_{0}^{\infty} \bar{L}\left(v(t), v^{\prime}(t)\right) \mathrm{d} t-\langle l, \bar{x}-x\rangle \geq \int_{0}^{\infty} \bar{L}\left(\Lambda^{(x)}(t),\left(\Lambda^{(x)}\right)^{\prime}(t)\right) \mathrm{d} t-\langle l, \bar{x}-x\rangle=\pi(x) .
$$

This completes the proof of Proposition 6.2.

Corollary 6.1. $\pi(\bar{x})=0$.

Proposition 6.3. There is $M_{*}>0$ such that for each $x \in \bar{X}_{*}$ satisfying $|x|>M_{*}$ the inequality $\pi(x) \geq 2$ holds.

Proof. By (2.1) there exists $M_{1}>0$ such that

$$
\psi\left(M_{1}\right)>a+|\bar{f}(\bar{x}, 0)|+4 .
$$

In view of Lemma 6.1 there exists $M_{*}>0$ such that if an a.c. function $v:[0,1] \rightarrow R^{n}$ satisfies

$$
\int_{0}^{1} \bar{f}\left(v(t), v^{\prime}(t)\right) \mathrm{d} t \leq(|l|+1)\left(|\bar{x}|+M_{1}+1\right)+|\bar{f}(\bar{x}, 0)|+4
$$


then

$$
|v(t)| \leq M_{*} \text { for all } t \in[0,1]
$$

Let

$$
x \in \bar{X}_{*} \text { and }|x|>M_{*} .
$$

Consider an $(\bar{f})$-overtaking optimal function $\Lambda^{(x)}:[0, \infty) \rightarrow R^{n}$ which is also $(\bar{f})$-good. It follows from $(6.11)$ and the choice of $M_{*}$ (see $\left.(6.10)\right)$ that

$$
\int_{0}^{1} \bar{f}\left(\Lambda^{(x)}(t),\left(\Lambda^{(x)}\right)^{\prime}(t)\right) \mathrm{d} t>(|l|+1)\left(|\bar{x}|+M_{1}+1\right)+|\bar{f}(\bar{x}, 0)|+4 .
$$

If $\left|\Lambda^{(x)}(t)\right|>M_{1}$ for each $t \geq 1$, then relations (3.3) and (6.9) imply that for each $t \geq 1$

$$
\bar{f}\left(\Lambda^{(x)}(t),\left(\Lambda^{(x)}\right)^{\prime}(t)\right)-\bar{f}(\bar{x}, 0) \geq \psi\left(\left|\Lambda^{(x)}(t)\right|\right)-a-\bar{f}(\bar{x}, 0) \geq 4
$$

and

\section{a contradiction.}

$$
\int_{0}^{T} \bar{f}\left(\Lambda^{(x)}(t),\left(\Lambda^{(x)}\right)^{\prime}(t)\right) \mathrm{d} t-T \bar{f}(\bar{x}, 0) \rightarrow \infty \text { as } T \rightarrow \infty
$$

Therefore there is $S_{0} \geq 1$ such that

$$
\left|\Lambda^{(x)}\left(S_{0}\right)\right| \leq M_{1},\left|\Lambda^{(x)}(t)\right|>M_{1} \text { for each } t \text { satisfying } 1 \leq t<S_{0} .
$$

It follows from $(3.3),(6.9)$ and (6.13) that

$$
\bar{f}\left(\Lambda^{(x)}(t),\left(\Lambda^{(x)}\right)^{\prime}(t)\right) \geq|\bar{f}(\bar{x}, 0)| \text { for each } t \text { such that } 1 \leq t<S_{0} .
$$

Then by (3.11), (3.10), (6.14), (3.5), (6.13), (6.12) and Proposition 2.3,

$$
\begin{aligned}
\pi(x)= & \int_{0}^{\infty} \bar{L}\left(\Lambda^{(x)}(t),\left(\Lambda^{(x)}\right)^{\prime}(t)\right) \mathrm{d} t-\langle l, \bar{x}-x\rangle \\
= & \lim _{T \rightarrow \infty} \int_{0}^{T}\left[\bar{f}\left(\Lambda^{(x)}(t),\left(\Lambda^{(x)}\right)^{\prime}(t)\right)-\bar{f}(\bar{x}, 0)\right] \mathrm{d} t \\
= & \int_{0}^{1}\left[\bar{f}\left(\Lambda^{(x)}(t),\left(\Lambda^{(x)}\right)^{\prime}(t)\right)\right] \mathrm{d} t-\bar{f}(\bar{x}, 0)+\int_{1}^{S_{0}}\left[\bar{f}\left(\Lambda^{(x)}(t),\left(\Lambda^{(x)}\right)^{\prime}(t)\right)-\bar{f}(\bar{x}, 0)\right] \mathrm{d} t \\
& +\lim _{T \rightarrow \infty} \int_{S_{0}}^{T}\left[\bar{f}\left(\Lambda^{(x)}(t),\left(\Lambda^{(x)}\right)^{\prime}(t)\right)-\bar{f}(\bar{x}, 0)\right] \mathrm{d} t \\
\geq & \int_{0}^{1} \bar{f}\left(\Lambda^{(x)}(t),\left(\Lambda^{(x)}\right)^{\prime}(t)\right) \mathrm{d} t-\bar{f}(\bar{x}, 0)+\lim _{T \rightarrow \infty} \int_{S_{0}}^{T} \bar{L}\left(\Lambda^{(x)}(t),\left(\Lambda^{(x)}\right)^{\prime}(t)\right) \mathrm{d} t \\
& -\lim _{T \rightarrow \infty}\left\langle l, \Lambda^{(x)}(T)-\Lambda^{(x)}\left(S_{0}\right)\right\rangle \\
\geq & \int_{0}^{1} \bar{f}\left(\Lambda^{(x)}(t),\left(\Lambda^{(x)}\right)^{\prime}(t)\right) \mathrm{d} t-\bar{f}(\bar{x}, 0)-|l|\left(|\bar{x}|+M_{1}\right)>4 .
\end{aligned}
$$

This completes the proof of Proposition 6.3.

Let $M_{*}>4$ be as guaranteed by Proposition 6.3. Namely

$$
\pi(x) \geq 2 \text { for each } x \in \bar{X}_{*} \text { satisfying }|x| \geq M_{*} .
$$


Proposition 6.4. There is $\tilde{M}>M_{*}$ such that for each $x \in \bar{X}_{*} \backslash \bar{X}_{\tilde{M}}$ the inequality $\pi(x) \geq 2$ holds.

Proof. By Proposition 4.2 there exists $\bar{M}>M_{*}$ such that for each $T>0$ and each a.c. function $v:[0, T] \rightarrow R^{n}$ which satisfies

$$
|v(0)| \leq M_{*}+1, I^{\bar{f}}(0, T, v) \leq T \bar{f}(\bar{x}, 0)+4
$$

the following inequality holds:

$$
|v(t)| \leq \bar{M} \text { for all } t \in[0, T]
$$

Choose a number

$$
\tilde{M}>M_{*}+\bar{M}+2+|l|\left(|\bar{x}|+M_{*}\right) 2 \bar{M}
$$

Let $x \in \bar{X}_{*} \backslash \bar{X}_{\tilde{M}}$. If $|x|>M_{*}$, then in view of (6.15) $\pi(x) \geq 2$. Assume that

$$
|x| \leq M_{*} .
$$

In order to complete the proof of the Proposition it is sufficient to show that $\pi(x) \geq 2$. Let us assume that

$$
\pi(x)<2 \text {. }
$$

Relations (3.10) and (3.11) imply that

$$
\pi(x)=\int_{0}^{\infty} \bar{L}\left(\Lambda^{(x)}(t),\left(\Lambda^{(x)}\right)^{\prime}(t)\right) \mathrm{d} t-\langle l, \bar{x}-x\rangle=\lim _{T \rightarrow \infty}\left[I^{\bar{f}}\left(0, T, \Lambda^{(x)}\right)-T f(\bar{x}, 0)\right] .
$$

By (6.20) and (6.21) for all large enough $T, I^{\bar{f}}\left(0, T, \Lambda^{(x)}\right) \leq T f(\bar{x}, 0)+2$. Combined with (6.19) and the choice of $\bar{M}$ (see (6.16) and (6.17)) this implies that

$$
\left|\Lambda^{(x)}(t)\right| \leq \bar{M} \text { for all } t \in[0, \infty) .
$$

It follows from (6.19)-(6.21) that

$$
\int_{0}^{\infty} \bar{L}\left(\Lambda^{(x)}(t),\left(\Lambda^{(x)}\right)^{\prime}(t)\right) \mathrm{d} t<2+|l|\left(|\bar{x}|+M_{*}\right) .
$$

Together with (3.5), (6.22) and (6.18) this inequality implies that for each $T>0$

$$
\begin{aligned}
I^{\bar{f}}\left(0, T, \Lambda^{(x)}\right)-T f(\bar{x}, 0) & =\int_{0}^{T} \bar{L}\left(\Lambda^{(x)}(t),\left(\Lambda^{(x)}\right)^{\prime}(t)\right) \mathrm{d} t-\left\langle l, \Lambda^{(x)}(T)-x\right\rangle \\
& <2+|l|\left(|\bar{x}|+M_{*}\right)+|l| 2 \bar{M}<\tilde{M} .
\end{aligned}
$$

By this inequality and (6.18) and (6.19) $x \in \bar{X}_{\tilde{M}}$, a contradiction. The contradiction we have reached proves that $\pi(x) \geq 2$. Proposition 6.5 is proved.

Proposition 6.5. For any $M>0$ the function $\pi: \bar{X}_{M} \rightarrow R^{1}$ is lower semicontinuous.

Proof. Let $M>0,\left\{x_{k}\right\}_{k=1}^{\infty} \subset \bar{X}_{M}, x \in \bar{X}_{M}$ and $\lim _{k \rightarrow \infty} x_{k}=x$. We will show that $\pi(x) \leq \liminf _{k \rightarrow \infty} \pi\left(x_{k}\right)$. We may assume that there exists a finite $\lim _{k \rightarrow \infty} \pi\left(x_{k}\right)$. By (3.10) and (3.11) for each integer $k \geq 1$

$$
\begin{aligned}
\pi\left(x_{k}\right) & =\int_{0}^{\infty} \bar{L}\left(\Lambda^{\left(x_{k}\right)}(t),\left(\Lambda^{\left(x_{k}\right)}\right)^{\prime}(t)\right) \mathrm{d} t-\left\langle l, \bar{x}-x_{k}\right\rangle \\
& =\lim _{T \rightarrow \infty}\left[I^{\bar{f}}\left(0, T, \Lambda^{\left(x_{k}\right)}\right)-T f(\bar{x}, 0)\right] .
\end{aligned}
$$


We have

$$
|x| \leq M \text { and }\left|x_{k}\right| \leq M \text { for all integers } k \geq 1 .
$$

Let $k \geq 1$ be an integer. Since $x_{k} \in \bar{X}_{M}$ there exists an a.c. function $v:[0, \infty) \rightarrow R^{n}$ such that

$$
v(0)=x_{k}, I^{\bar{f}}(0, T, v)-T f(\bar{x}, 0) \leq M \text { for all } T>0 .
$$

Since the function $\Lambda^{\left(x_{k}\right)}$ is $(\bar{f})$-overtaking optimal (6.25) implies that

$$
\lim _{T \rightarrow \infty}\left[I^{\bar{f}}\left(0, T, \Lambda^{\left(x_{k}\right)}\right)-T f(\bar{x}, 0)\right]-M \leq \limsup _{T \rightarrow \infty}\left[I^{\bar{f}}\left(0, T, \Lambda^{\left(x_{k}\right)}\right)-T f(\bar{x}, 0)-\left(I^{f}(0, T, v)-T f(\bar{x}, 0)\right)\right] \leq 0
$$

Thus

$$
\lim _{T \rightarrow \infty}\left(I^{\bar{f}}\left(0, T, \Lambda^{\left(x_{k}\right)}\right)-T f(\bar{x}, 0)\right) \leq M \text { for all integers } k \geq 1 .
$$

In view of Proposition 4.2, (6.24) and (6.26) there exists $M_{0}>0$ such that

$$
\left|\Lambda^{\left(x_{k}\right)}(t)\right| \leq M_{0} \text { for all } t \in[0, \infty) \text { and all integers } k \geq 1
$$

It follows from (6.26), (6.27) and Proposition 2.1 that there exists $M_{1}>0$ such that for each integer $k \geq 1$ and each $T>0$,

$$
I^{\bar{f}}\left(0, T, \Lambda^{\left(x_{k}\right)}\right) \leq T f(\bar{x}, 0)+M_{1}
$$

By (6.27), (6.28) and Proposition 4.1 there exists a strictly increasing sequence of natural numbers $\left\{k_{i}\right\}_{i=1}^{\infty}$ and an a.c. function $u:[0, \infty) \rightarrow R^{n}$ such that for each integer $m \geq 1$,

$$
\begin{gathered}
\Lambda^{\left(x_{k_{i}}\right)}(t) \rightarrow u(t) \text { as } i \rightarrow \infty \text { uniformly on }[0, m] \\
I^{\bar{f}}(0, m, u) \leq \liminf _{i \rightarrow \infty} I^{\bar{f}}\left(0, m, \Lambda^{\left(x_{k_{i}}\right)}\right)
\end{gathered}
$$

Relation (6.29) implies that

$$
u(0)=\lim _{i \rightarrow \infty} \Lambda^{\left(x_{k_{i}}\right)}(0)=\lim _{i \rightarrow \infty} x_{k_{i}}=x .
$$

In view of (3.5), (3.11) and (6.29) for each integer $m \geq 1$

$$
\begin{aligned}
\int_{0}^{m} \bar{L}\left(u(t), u^{\prime}(t)\right) \mathrm{d} t & =\int_{0}^{m} \bar{f}\left(u(t), u^{\prime}(t)\right) \mathrm{d} t-m \bar{f}(\bar{x}, 0)+\langle l, u(m)-u(0)\rangle \\
& \leq \liminf _{i \rightarrow \infty}\left[I^{\bar{f}}\left(0, m, \Lambda^{\left(x_{k_{i}}\right)}\right)-m \bar{f}(\bar{x}, 0)+\left\langle l, \Lambda^{\left(x_{k_{i}}\right)}(m)-\Lambda^{\left(x_{k_{i}}\right)}(0)\right\rangle\right] \\
& =\liminf _{i \rightarrow \infty} \int_{0}^{m} \bar{L}\left(\Lambda^{\left(x_{k_{i}}\right)}(t),\left(\Lambda^{\left(x_{k_{i}}\right)}\right)^{\prime}(t)\right) \mathrm{d} t \leq \liminf _{i \rightarrow \infty} \int_{0}^{\infty} \bar{L}\left(\Lambda^{\left(x_{k_{i}}\right)}(t),\left(\Lambda^{\left(x_{k_{i}}\right)}\right)^{\prime}(t)\right) \mathrm{d} t \\
& \leq \liminf _{i \rightarrow \infty}\left[\pi\left(x_{k_{i}}\right)+\left\langle l, \bar{x}-x_{k_{i}}\right\rangle\right]=\liminf _{i \rightarrow \infty} \pi\left(x_{k_{i}}\right)+\langle l, \bar{x}-x\rangle .
\end{aligned}
$$

This implies that

$$
\int_{0}^{\infty} \bar{L}\left(u(t), u^{\prime}(t)\right) \mathrm{d} t-\langle l, \bar{x}-x\rangle \leq \liminf _{i \rightarrow \infty} \pi\left(x_{k_{i}}\right) .
$$

Combined with Proposition 6.2 this implies that $\pi(x) \leq \liminf _{i \rightarrow \infty} \pi\left(x_{k_{i}}\right)$. Proposition 6.6 is proved.

Proposition 6.6. Let $y, z \in \bar{X}_{*}, y \neq z$ and let $\alpha \in(0,1)$. Then $\pi(\alpha y+(1-\alpha) z)<\alpha \pi(y)+(1-\alpha) \pi(z)$. 
Proof. Since $y, z \in \bar{X}_{*}$ the functions $\Lambda^{(y)}$ and $\Lambda^{(z)}$ are $(\bar{f})$-good. In view of Proposition 2.2 the function $\alpha \Lambda^{(y)}+(1-\alpha) \Lambda^{(z)}$ is also $\bar{f}$-good. Since the integrand $\bar{L}$ is convex we have that for all $t \geq 0$

$$
\begin{aligned}
\bar{L}\left(\alpha \Lambda^{(y)}(t)+(1-\alpha) \Lambda^{(z)}(t), \alpha\left(\Lambda^{(y)}\right)^{\prime}(t)+(1-\alpha)\left(\Lambda^{(z)}\right)^{\prime}(t)\right) & \leq \\
& \alpha \bar{L}\left(\Lambda^{(y)}(t),\left(\Lambda^{(y)}\right)^{\prime}(t)\right)+(1-\alpha) \bar{L}\left(\Lambda^{(z)}(t),\left(\Lambda^{(z)}\right)^{\prime}(t)\right) .
\end{aligned}
$$

The relation $y \neq z$ implies that for all positive $t$ which are close enough to zero, $\Lambda^{(y)}(t) \neq \Lambda^{(z)}(t)$ and

$$
\begin{aligned}
\bar{L}\left(\alpha \Lambda^{(y)}(t)+(1-\alpha) \Lambda^{(z)}(t), \alpha\left(\Lambda^{(y)}\right)^{\prime}(t)+(1-\alpha)\left(\Lambda^{(z)}\right)^{\prime}(t)\right) & < \\
& \alpha \bar{L}\left(\Lambda^{(y)}(t),\left(\Lambda^{(y)}\right)^{\prime}(t)\right)+(1-\alpha) \bar{L}\left(\Lambda^{(z)}(t),\left(\Lambda^{(z)}\right)^{\prime}(t)\right) .
\end{aligned}
$$

The inequalities above imply that

$$
\begin{aligned}
\left.\int_{0}^{\infty} \bar{L}\left(\alpha \Lambda^{(y)}(t)+(1-\alpha) \Lambda^{(z)}(t)\right), \alpha\left(\Lambda^{(y)}\right)^{\prime}(t)+(1-\alpha)\left(\Lambda^{(z)}\right)^{\prime}(t)\right) \mathrm{d} t< \\
\quad \alpha \int_{0}^{\infty} \bar{L}\left(\Lambda^{(y)}(t),\left(\Lambda^{(y)}\right)^{\prime}(t)\right) \mathrm{d} t+(1-\alpha) \int_{0}^{\infty} \bar{L}\left(\Lambda^{(z)}(t),\left(\Lambda^{(z)}\right)^{\prime}(t)\right) \mathrm{d} t .
\end{aligned}
$$

By (6.31), Proposition 6.2 and (3.11)

$$
\begin{aligned}
\pi(\alpha y+(1-\alpha) z) \leq & \int_{0}^{\infty} \bar{L}\left(\alpha \Lambda^{(y)}(t)+(1-\alpha) \Lambda^{(z)}(t), \alpha\left(\Lambda^{(y)}\right)^{\prime}(t)+(1-\alpha)\left(\Lambda^{(z)}\right)^{\prime}(t)\right) \mathrm{d} t \\
& -\langle l, \bar{x}-\alpha y-(1-\alpha) z\rangle<\alpha\left[\int_{0}^{\infty} \bar{L}\left(\Lambda^{(y)}(t),\left(\Lambda^{(y)}\right)^{\prime}(t)\right) \mathrm{d} t-\langle l, \bar{x}-y\rangle\right] \\
& +(1-\alpha)\left[\int_{0}^{\infty} \bar{L}\left(\Lambda^{(z)}(t),\left(\Lambda^{(z)}\right)^{\prime}(t)\right) \mathrm{d} t-\langle l, \bar{x}-z\rangle\right] \\
= & \alpha \pi(y)+(1-\alpha) \pi(z) .
\end{aligned}
$$

Proposition 6.6 is proved.

Now Proposition 3.1 follows from Propositions 6.4-6.6 and Corollary 4.1.

\section{Proof of Theorem 3.3}

Let $v:[0, T] \rightarrow R^{n}$ be an a.c. function. Put

$$
\bar{v}(t)=v(T-t), t \in[0, T] .
$$

It is clear that

$$
\begin{aligned}
\int_{0}^{T} \bar{f}\left(\bar{v}(t), \bar{v}^{\prime}(t)\right) \mathrm{d} t & =\int_{0}^{T} f\left(\bar{v}(t),-\bar{v}^{\prime}(t)\right) \mathrm{d} t \\
& =\int_{0}^{T} f\left(v(T-t), v^{\prime}(T-t)\right) \mathrm{d} t=\int_{0}^{T} f\left(v(t), v^{\prime}(t)\right) \mathrm{d} t .
\end{aligned}
$$

The following lemma is an important ingredient in the proof of Theorem 3.3. 
Lemma 7.1. Let $\epsilon, M$ be a pair of positive numbers and let $L_{1}$ be a natural number. Then there exist a natural number $L_{2}$ and $\delta>0$ such that for each $T \geq L_{2}$ and each a.c. function $v:[0, T] \rightarrow R^{n}$ which satisfies

$$
|v(0)| \leq M, \int_{0}^{T} \bar{L}\left(v(t), v^{\prime}(t)\right) \mathrm{d} t+\langle l,-\bar{x}+v(0)\rangle \leq \pi\left(x_{*}\right)+\delta
$$

the inequality $\left|v(t)-\Lambda^{\left(x_{*}\right)}(t)\right| \leq \epsilon$ holds for all $t \in\left[0, L_{1}\right]$.

Proof. Let us assume the contrary. Then for each natural number $k$ there exist $T_{k} \geq k$ and an a.c. function $v_{k}:\left[0, T_{k}\right] \rightarrow R^{n}$ such that

$$
\begin{aligned}
M \geq\left|v_{k}(0)\right|, & \int_{0}^{T_{k}} \bar{L}\left(v_{k}(t), v_{k}^{\prime}(t)\right) \mathrm{d} t-\left\langle l, \bar{x}-v_{k}(0)\right\rangle \leq \pi\left(x_{*}\right)+k^{-1}, \\
& \sup \left\{\left|v_{k}(t)-\Lambda^{\left(x^{*}\right)}(t)\right|: t \in\left[0, L_{1}\right]\right\}>\epsilon .
\end{aligned}
$$

We will show that there exists a subsequence $\left\{v_{k_{i}}\right\}_{i=1}^{\infty}$ which converges uniformly on any bounded subinterval of $[0, \infty)$ to an $(\bar{f})$-overtaking optimal function $v$ satisfying $v(0)=x_{*}$.

By Proposition 4.2 (with $f=\bar{L}$ ) and (7.2) there is $M_{0}>0$ such that

$$
\left|v_{k}(t)\right| \leq M_{0} \text { for all } t \in\left[0, T_{k}\right] \text { and all integers } k \geq 1
$$

In view of Proposition 4.2 (with $f=\bar{L}$ ), (7.2) and (7.4) there exist a strictly increasing sequence of natural numbers $\left\{k_{i}\right\}_{i=1}^{\infty}$ and an a.c. function $v:[0, \infty) \rightarrow R^{n}$ such that for each integer $m \geq 1$

$$
\begin{gathered}
v_{k_{i}}(t) \rightarrow v(t) \text { as } i \rightarrow \infty \text { uniformly on }[0, m], \\
\int_{0}^{m} \bar{L}\left(v(t), v^{\prime}(t)\right) \mathrm{d} t \leq \liminf _{i \rightarrow \infty} \int_{0}^{m} \bar{L}\left(v_{k_{i}}(t), v_{k_{i}}^{\prime}(t)\right) \mathrm{d} t .
\end{gathered}
$$

Relations (7.2) and (7.5) imply that

$$
|v(0)| \leq M
$$

It follows from (7.2) and (7.5) that for each integer $m \geq 1$,

$$
\begin{aligned}
\int_{0}^{m} \bar{L}\left(v(t), v^{\prime}(t)\right) \mathrm{d} t & \leq \liminf _{i \rightarrow \infty} \int_{0}^{T_{k_{i}}} \bar{L}\left(v_{k_{i}}(t), v_{k_{i}}^{\prime}(t)\right) \mathrm{d} t \\
& \leq \liminf _{i \rightarrow \infty}\left[\pi\left(x_{*}\right)+k_{i}^{-1}+\left\langle l, \bar{x}-v_{k_{i}}(0)\right\rangle\right]=\pi\left(x_{*}\right)+\langle l, \bar{x}-v(0)\rangle .
\end{aligned}
$$

This implies that

$$
\int_{0}^{\infty} \bar{L}\left(v(t), v^{\prime}(t)\right) \mathrm{d} t+\langle l, v(0)-\bar{x}\rangle \leq \pi\left(x_{*}\right)
$$

By (7.7) and Proposition 6.1, $v$ is an $(\bar{f})$-good function. Proposition 6.2 and (7.7) imply that

$$
\pi(v(0)) \leq \int_{0}^{\infty} \bar{L}\left(v(t), v^{\prime}(t)\right) \mathrm{d} t-\langle l, \bar{x}-v(0)\rangle \leq \pi\left(x_{*}\right) .
$$

In view of the choice of $x_{*}$ (see (3.12) and (3.13)), (3.5) and Proposition 2.3

$$
\begin{gathered}
v(0)=x_{*}, \\
\pi\left(x_{*}\right)=\int_{0}^{\infty} \bar{L}\left(v(t), v^{\prime}(t)\right) \mathrm{d} t-\left\langle l, \bar{x}-x_{*}\right\rangle=\lim _{T \rightarrow \infty}\left[I^{\bar{f}}(0, T, v)-T f(\bar{x}, 0)\right] .
\end{gathered}
$$


It follows from (7.8), (3.10) and (3.11) that $v$ is an $(\bar{f})$-overtaking optimal function. Together with Theorem 3.1 this implies that

$$
v(t)=\Lambda^{\left(x_{*}\right)}(t) \text { for all } t \in[0, \infty) .
$$

By (7.5) and (7.9) for each sufficiently large integers $i \geq 1$,

$$
\sup \left\{\left|v_{k_{i}}(t)-\Lambda^{\left(x_{*}\right)}(t)\right|: t \in\left[0, L_{1}\right]\right\}<\epsilon / 2 .
$$

This contradicts (7.3). The contradiction we have reached proves Lemma 7.1.

Proof of Theorem 3.3. We will choose the constants $\delta$ and $L_{2}$. First we choose $\bar{r} \in\left(0,4^{-1}\right)$ such that

$$
\left\{(x, y) \in R^{n} \times R^{n}:|x-\bar{x}| \leq 4 \bar{r},|y| \leq 4 \bar{r}\right\} \subset \operatorname{dom}(f) .
$$

By Proposition 4.2 there is $M_{0}>M$ such that for each $T>0$ and each a.c. function $u:[0, T] \rightarrow R^{n}$ which satisfies

the following inequality holds:

$$
|u(0)| \leq M, I^{f}(0, T, u) \leq T f(\bar{x}, 0)+M+2
$$

$$
|u(t)| \leq M_{0}, t \in[0, T]
$$

Lemma 7.1 implies that there exist a natural number $L_{12}>L_{1}$ and $\delta_{0}>0$ such that the following property holds:

(P1) For each $T \geq L_{12}$ and each a.c. function $u:[0, T] \rightarrow R^{n}$ satisfying

$$
|u(0)| \leq M_{0}, \int_{0}^{T} \bar{L}\left(u(t), u^{\prime}(t)\right) \mathrm{d} t+\langle l,-\bar{x}+u(0)\rangle \leq \pi\left(x_{*}\right)+\delta_{0}
$$

the inequality $\left|u(t)-\Lambda^{\left(x_{*}\right)}(t)\right| \leq \epsilon$ holds for all $t \in\left[0, L_{1}\right]$.

In view of Proposition 4.3 there exists $\delta_{1} \in(0,1)$ such that for each a.c. function $u:[0,1] \rightarrow R^{n}$ satisfying

$$
|u(0)-\bar{x}|,|u(1)-\bar{x}| \leq \delta_{1}
$$

the inequality

$$
I^{f}(0,1, u) \geq f(\bar{x}, 0)-\delta_{0} / 16
$$

holds. Since $f$ is continuous at $(\bar{x}, 0)$ there exists a positive number $\delta_{2}$ such that:

$$
\begin{gathered}
\left|f\left(\xi_{1}, \xi_{2}\right)-f(\bar{x}, 0)\right| \leq \delta_{0} / 16 \text { for all }\left(\xi_{1}, \xi_{2}\right) \in R^{n} \times R^{n} \text { satisfying } \\
\qquad \xi_{1}-\bar{x}\left|\leq 2 \delta_{2},\right| \xi_{2} \mid \leq 2 \delta_{2} ; \\
2(|l|+1) \delta_{2}<\delta_{0} / 16 \text { and } \delta_{2}<\min \left\{\bar{r}, \delta_{1}\right\} .
\end{gathered}
$$

By Theorem 2.2 there exist an integer $L_{13}>L_{12}$ and a positive number $\delta<\delta_{2}$ such that the following property holds:

(P2) If a number $T>2 L_{13}$ and if an a.c. function $u:[0, T] \rightarrow R^{n}$ satisfies

$$
u(0) \in X_{M}, I^{f}(0, T, u) \leq \sigma(f, T, u(0))+\delta,
$$

then

$$
|u(t)-\bar{x}| \leq \delta_{2}, t \in\left[L_{13}, T-L_{13}\right] .
$$

In view of Proposition 2.3 there is a natural number $L_{2}>L_{13}$ such that

$$
\left|\Lambda^{\left(x_{*}\right)}(t)-\bar{x}\right| \leq \delta_{2} \text { for all numbers } t \geq L_{2} .
$$


Thus we have chosen the constants $L_{2}$ and $\delta$.

Let $T>2 L_{2}$ and let an a.c. function $v:[0, T] \rightarrow R^{n}$ satisfies

$$
v(0) \in X_{M}, I^{f}(0, T, v) \leq \sigma(f, T, v(0))+\delta .
$$

We will show that

$$
\left|v(T-t)-\Lambda^{(*)}(t)\right| \leq \epsilon \text { for all } t \in\left[0, L_{1}\right]
$$

It follows from (7.17) and the definition of $X_{M}$ that there is an a.c. function $u:[0, \infty) \rightarrow R^{n}$ such that

$$
u(0)=v(0) \text { and } I^{f}(0, T, u)-T f(\bar{x}, 0) \leq M \text { for all } T>0 .
$$

Relations (7.17) and (7.19) imply that

$$
I^{f}(0, T, v) \leq I^{f}(0, T, u)+\delta \leq T f(\bar{x}, 0)+M+1 .
$$

Together with the choice of $M_{0}$ (see (7.11) and (7.12)) and (7.17) this implies that

$$
|v(t)| \leq M_{0} \text { for all } t \in[0, T]
$$

By the property (P2) and (7.17),

$$
|v(t)-\bar{x}| \leq \delta_{2} \text { for all } t \in\left[L_{13}, T-L_{13}\right]
$$

Define

$$
\begin{gathered}
y(t)=v(t), t \in\left[0, T-L_{2}-1\right], y(t)=\Lambda^{\left(x_{*}\right)}(T-t), t \in\left[T-L_{2}, T\right], \\
y(t)=v\left(T-L_{2}-1\right)+\left(t-\left(T-L_{2}-1\right)\right)\left[\Lambda^{\left(x_{*}\right)}\left(L_{2}\right)-v\left(T-L_{2}-1\right)\right], \\
t \in\left(T-L_{2}-1, T-L_{2}\right) .
\end{gathered}
$$

Relations (7.16), (7.22) and (7.23) imply that for all $t \in\left[T-L_{2}-1, T-L_{2}\right]$,

$$
|y(t)-\bar{x}| \leq \max \left\{\left|v\left(T-L_{2}-1\right)-\bar{x}\right|,\left|\Lambda^{\left(x_{*}\right)}\left(L_{2}\right)-\bar{x}\right|\right\} \leq \delta_{2},\left|y^{\prime}(t)\right| \leq 2 \delta_{2} .
$$

By (7.24) and the choice of $\delta_{2}($ see $(7.14))$ for all $t \in\left[T-L_{2}-1, T-L_{2}\right]$,

$$
\begin{gathered}
\left|f\left(y(t), y^{\prime}(t)\right)-f(\bar{x}, 0)\right| \leq \delta_{0} / 16, \\
\left|I^{f}\left(T-L_{2}-1, T-L_{2}, y\right)-f(\bar{x}, 0)\right| \leq \delta_{0} / 16 .
\end{gathered}
$$

In view of (7.22) and the choice of $\delta_{1}($ see $(7.13)) I^{f}\left(T-L_{2}-1, T-L_{2}, v\right) \geq f(\bar{x}, 0)-\delta_{0} / 16$. It follows from this inequality, (7.17) and (7.23) that

$$
\begin{aligned}
\delta \geq & I^{f}(0, T, v)-I^{f}(0, T, y) \\
= & I^{f}\left(0, T-L_{2}-1, v\right)+I^{f}\left(T-L_{2}-1, T-L_{2}, v\right)+I^{f}\left(T-L_{2}, T, v\right) \\
& \quad-I^{f}\left(0, T-L_{2}-1, y\right)-I^{f}\left(T-L_{2}-1, T-L_{2}, y\right)-I^{f}\left(T-L_{2}, T, y\right) \\
\geq & f(\bar{x}, 0)-\delta_{0} / 16-\left(f(\bar{x}, 0)+\delta_{0} / 16\right) \\
& \quad+I^{f}\left(T-L_{2}, T, v\right)-I^{f}\left(T-L_{2}, T, y\right) \\
\geq & -\delta_{0} / 8+\int_{T-L_{2}}^{T} f\left(v(t), v^{\prime}(t)\right) \mathrm{d} t-\int_{0}^{L_{2}} \bar{f}\left(\Lambda^{\left(x_{*}\right)}(t),\left(\Lambda^{\left(x_{*}\right)}\right)^{\prime}(t)\right) \mathrm{d} t .
\end{aligned}
$$


Set

By (7.15), (7.26), (3.5), (7.16) and (7.22),

$$
\bar{v}(t)=v(T-t), t \in\left[0, L_{2}\right] .
$$

$$
\begin{gathered}
\delta_{0} / 4 \geq \delta+\delta_{0} / 8 \geq \int_{0}^{L_{2}} \bar{f}\left(\bar{v}(t), \bar{v}^{\prime}(t)\right) \mathrm{d} t-\int_{0}^{L_{2}} \bar{f}\left(\Lambda^{\left(x_{*}\right)}(t),\left(\Lambda^{\left(x_{*}\right)}\right)^{\prime}(t)\right) \mathrm{d} t \\
=\int_{0}^{L_{2}} \bar{L}\left(\bar{v}(t), \bar{v}^{\prime}(t)\right) \mathrm{d} t-\left\langle l, \bar{v}\left(L_{2}\right)-\bar{v}(0)\right\rangle \\
-\left[\int_{0}^{L_{2}} \bar{L}\left(\Lambda^{\left(x_{*}\right)}(t),\left(\Lambda^{\left(x_{*}\right)}\right)^{\prime}(t)\right) \mathrm{d} t-\left\langle l, \Lambda^{\left(x_{*}\right)}\left(L_{2}\right)-\Lambda^{\left(x_{*}\right)}(0)\right\rangle\right] \\
\geq \int_{0}^{L_{2}} \bar{L}\left(\bar{v}(t), \bar{v}^{\prime}(t)\right) \mathrm{d} t-\langle l, \bar{x}-\bar{v}(0)\rangle \\
-\left[\int_{0}^{L_{2}} \bar{L}\left(\Lambda^{\left(x_{*}\right)}(t),\left(\Lambda^{\left(x_{*}\right)}\right)^{\prime}(t)\right) \mathrm{d} t-\left\langle l, \bar{x}-\Lambda^{\left(x_{*}\right)}(0)\right\rangle\right]-2 \delta_{2}|l| .
\end{gathered}
$$

Together with (7.15) and (3.11) this implies that

$$
\begin{gathered}
\left.\int_{0}^{L_{2}} \bar{L}\left(\bar{v}(t), \bar{v}^{\prime}(t)\right) \mathrm{d} t-\langle l, \bar{x}-\bar{v}(0)\rangle \leq \delta_{0} / 4+\delta_{0} / 8+\int_{0}^{\infty} \bar{L}\left(\Lambda^{\left(x_{*}\right)}(t),\left(\Lambda^{\left(x_{*}\right)}\right)^{\prime}(t)\right) \mathrm{d} t-\left\langle l, \bar{x}-\Lambda^{\left(x_{*}\right)}(0)\right\rangle\right] \\
\leq \delta_{0} / 2+\pi\left(x_{*}\right) .
\end{gathered}
$$

It follows from $(\mathrm{P} 1),(7.21),(7.27)$ and (7.28) that $\left|\bar{v}(t)-\Lambda^{\left(x_{*}\right)}(t)\right| \leq \epsilon$ for all $t \in\left[0, L_{1}\right]$ and $\left|v(T-t)-\Lambda^{\left(x_{*}\right)}(t)\right| \leq \epsilon$ for all $t \in\left[0, L_{1}\right]$. This completes the proof of Theorem 3.3.

\section{REFERENCES}

[1] H. Atsumi, Neoclassical growth and the efficient program of capital accumulation. Rev. Econ. Studies 32 (1965) 127-136.

[2] L. Cesari, Optimization - theory and applications. Springer-Verlag, New York (1983).

[3] D. Gale, On optimal development in a multi-sector economy. Rev. Econ. Studies 34 (1967) 1-18.

[4] M. Giaquinta and E. Guisti, On the regularity of the minima of variational integrals. Acta Math. 148 (1982) 31-46.

[5] A. Leizarowitz, Infinite horizon autonomous systems with unbounded cost. Appl. Math. Opt. 13 (1985) 19-43.

[6] A. Leizarowitz and V.J. Mizel, One dimensional infinite horizon variational problems arising in continuum mechanics. Arch. Rational Mech. Anal. 106 (1989) 161-194.

[7] M. Marcus and A.J. Zaslavski, The structure of extremals of a class of second order variational problems. Ann. Inst. H. Poincaré Anal. Non Linéaire 16 (1999) 593-629.

[8] L.W. McKenzie Classical general equilibrium theory. The MIT press, Cambridge, Massachusetts, USA (2002).

[9] J. Moser, Minimal solutions of variational problems on a torus. Ann. Inst. H. Poincaré Anal. Non Linéaire 3 (1986) $229-272$.

[10] P.H. Rabinowitz and E. Stredulinsky, On some results of Moser and of Bangert. Ann. Inst. H. Poincaré Anal. Non Linéaire 21 (2004) 673-688.

[11] P.H. Rabinowitz and E. Stredulinsky, On some results of Moser and of Bangert. II. Adv. Nonlinear Stud. 4 (2004) 377-396.

[12] R.T. Rockafellar, Convex analysis. Princeton University Press, Princeton, USA (1970).

[13] P.A. Samuelson, A catenary turnpike theorem involving consumption and the golden rule. Am. Econ. Rev. 55 (1965) $486-496$.

[14] C.C. von Weizsacker, Existence of optimal programs of accumulation for an infinite horizon. Rev. Econ. Studies 32 (1965) $85-104$.

[15] A.J. Zaslavski, Optimal programs on infinite horizon 1. SIAM J. Contr. Opt. 33 (1995) 1643-1660.

[16] A.J. Zaslavski, Optimal programs on infinite horizon 2. SIAM J. Contr. Opt. 33 (1995) 1661-1686.

[17] A.J. Zaslavski, Turnpike properties in the calculus of variations and optimal control. Springer, New York (2006).

[18] A.J. Zaslavski, Structure of extremals of autonomous convex variational problems. Nonlinear Anal. Real World Appl. 8 (2007) 1186-1207.

[19] A.J. Zaslavski, A turnpike result for a class of problems of the calculus of variations with extended-valued integrands. J. Convex Analysis (to appear). 